Large scale radial stability density of Hill's equation

This article has been downloaded from IOPscience. Please scroll down to see the full text article.

2013 Nonlinearity 26565

(http://iopscience.iop.org/0951-7715/26/2/565)

View the table of contents for this issue, or go to the journal homepage for more

Download details:

IP Address: 129.125.51.104

The article was downloaded on 10/01/2013 at 13:45

Please note that terms and conditions apply. 


\title{
Large scale radial stability density of Hill's equation
}

\author{
Henk Broer ${ }^{1}$, Mark Levi ${ }^{2}$ and Carles Simo ${ }^{3}$ \\ ${ }^{1}$ Johann Bernoulli Institute for Mathematics and Computer Science, University of Groningen, \\ Nijenborgh 9, 9747 AC Groningen, The Netherlands \\ 2 Department of Mathematics, Pennsylvania State University, University Park, PA, USA \\ ${ }^{3}$ Departament de Matemàtica Aplicada i Anàlisi, Gran Via 585, 08007 Barcelona, Spain
}

Received 10 September 2012, in final form 30 November 2012

Published 10 January 2013

Online at stacks.iop.org/Non/26/565

Recommended by A Chenciner

\begin{abstract}
This paper deals with large scale aspects of Hill's equation $\ddot{x}+(a+b p(t)) x=0$, where $p$ is periodic with a fixed period. In particular, the interest is the asymptotic radial density of the stability domain in the $(a, b)$-plane. It turns out that this density changes discontinuously in a certain direction and exhibits and interesting asymptotic fine structure. Most of the paper deals with the case where $p$ is a Morse function with one maximum and one minimum, but also the discontinuous case of square Hill's equation is studied, where the density behaves differently.
\end{abstract}

Mathematics Subject Classification: 34C08, 34D23, 26B15, 54C30

\section{Introduction, main results}

One of the simplest conservative systems, used to describe many vibratory motions, is given by the Mathieu equations [14]

$$
\ddot{x}+(a+b \cos (t)) x=0,
$$

where $a$ and $b$ are real parameters. One of the important features of equation (1) is that of parametric resonance. Indeed, in the $(a, b)$-parameter plane zones emerge from the points $(a, b)=\left((k / 2)^{2}, 0\right), k=1,2, \ldots$, where the zero-solution becomes unstable, while outside these zones this solution is stable. Compare with Arnold [5] and see figure 1. We also note that the Mathieu equation is reversible and also that its potential is antisymmetric with respect to $t=\pi / 2$. Hence the stability diagram is symmetric with respect to the $a$-axis.

This paper deals with the more general class of Hill's equations

$$
\ddot{x}+(a+b p(t)) x=0, \quad p(t) \equiv p(t+1),
$$

where again $a$ and $b$ are real parameters. In many examples the period will be $2 \pi$ or some other fixed value, but this is irrelevant. Throughout we also assume that $p$ has average zero, i.e. that $\int_{0}^{1} p(t) \mathrm{d} t=0$. Parametric resonance occurs also in (2) and the parameter plane is divided into 

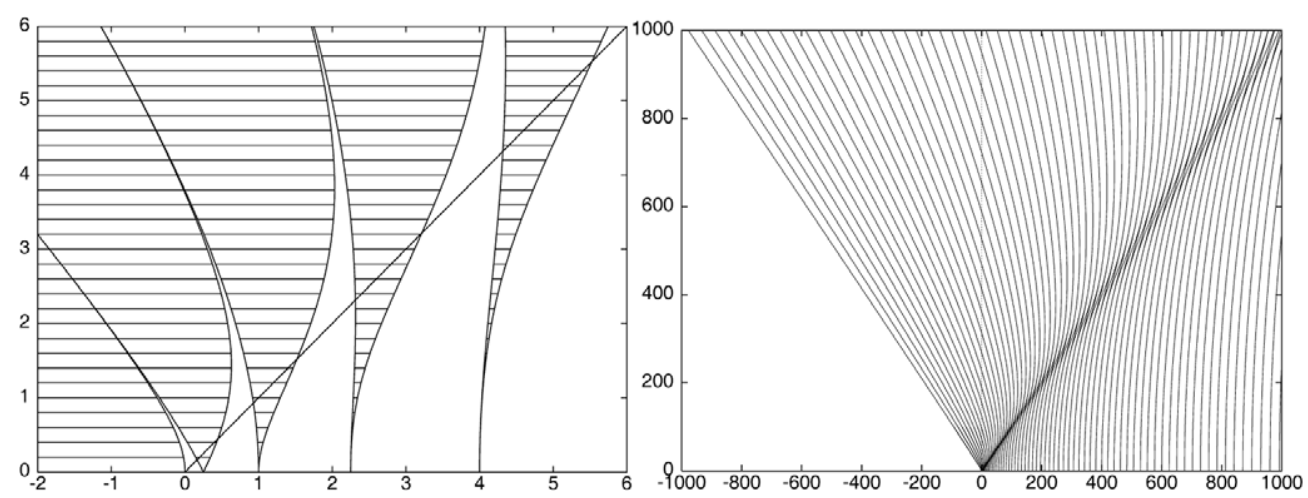

Figure 1. Stability diagram of Mathieu equation (1) depicted in the $(a, b)$-plane. Left: local aspects for small $b>0$. The shaded regions are part of the domain of unstability, where the zero-solution is hyperbolic. The line with slope $1, b=a$, is the critical line, to be introduced later, in present case. Right: a more global plot; here and later no shadings are being applied.

stable and unstable zones. Hill's equation arises as the variational equation of periodic solutions in Hamiltonian systems with two degrees of freedom and therefore has a wide applicability in all physical sciences. Since (2) gives a precise indication on the linear stability of these solutions it is worthwile to study this equation and its stability diagram. For a local study as $b \rightarrow 0$ we refer to [9] and references therein. We note that the boundaries between the stable and unstable zones form a bifurcation diagram, mainly related to subharmonic bifurcations.

The first stability diagram known to us is by van der Pol and Strutt [16]. Subsequently, many papers and textbooks have appeared on this subject; we would like to mention Stoker [20], Meixner and Schäfke [15], Levy and Keller [12], Weinstein and Keller [21, 22], Pöschel and Trubowitz [17] and Arnold [3, 4, 5]. This literature contains extensive estimates on the order of the tangencies of the instability boundaries for $b \rightarrow 0$, the so-called resonance tongues, as well as of the order of narrowness of the stability boundaries for $(-a, b) \rightarrow \infty$. It should be noted that such (Arnold) resonance tongues occur quite universally in the theory of dynamical systems and their bifurcations, which usually are successfully approached by averaging or normal form techniques [4], also see [7], and references therein.

In $[2,8,9]$ the topology of the symplectic group $\operatorname{Sp}(1, \mathbb{R}) \equiv S L(2, \mathbb{R})$ has been used to explain certain geometrical aspects of the resonance tongues of the reversible Hill equation, in particular the occurrence of so-called instability pockets. Similar results also hold in the case of Hill's equation with quasi-periodic forcing, which has direct applications to the quasi-periodic Schrödinger operator $[10,18,19]$. We would like to note that in this paper no reversibility will be assumed. However, as far as the authors know, the phenomena reported in this paper and the corresponding theoretical results are novel. Figure 2 shows a sample of examples concerning the global bifurcation diagram for different choices for the function $p$ in (2). These examples are reversible: $p(-t)=p(t)$. In figure 3 one can see a non-reversible example in a very large domain, both for $b>0$ and $b<0$. This paper focuses on certain global aspects of this bifurcation diagram regarding the large scale radial density of the stability domain.

To be more precise, under the regularity assumption that $t \mapsto a+b p(t)$ is a Morse function, we study the asymptotic measure theoretical density of the stability domain in the parameter plane, i.e. the set of $(a, b)$ for which the zero-solution of equation (2) is stable. In this paper a Morse function is understood as being smooth without degenerate critical points. Here the density is measured in radial directions. It is shown that this density equals 1 inside a certain sector, so here for 'most' parameter points one has stability. Outside this sector the density 

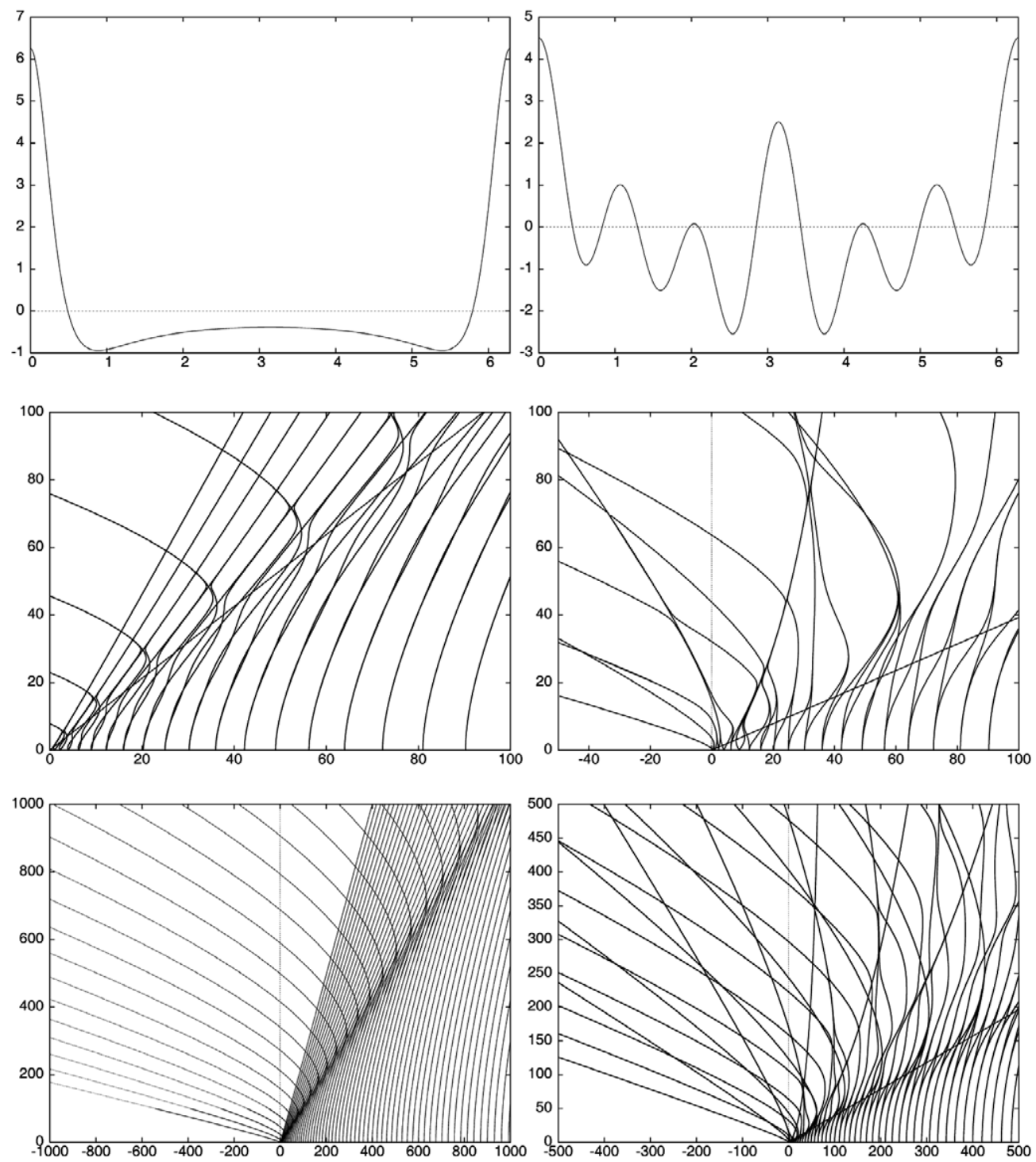

Figure 2. Bifurcation diagrams of the reversible Hill equation (2) for two choices of the function $p$. Left: Ince equation $p(t)=\left(1 /\left(1+\mu^{2}\right)\right)(\cos (t)-v) /\left((1-v \cos (t))^{2}\right), v=\left(2 \mu / 1+\mu^{2}\right)$, for the value $\mu=0.6$. Right: $p(t)=\cos (t)+\cos (2 t)+\cos (4 t)+1.5 \cos (6 t)$. In both cases we plotted two diagrams at different scale, in which we indicated the critical lines, except in the bottom left plot were it is obvious where the critical line is located. Also in each case we plotted on top the function $t \mapsto p(t)$.

equals 0 , so here for 'most' parameter points equation (2) is unstable. The critical direction occurs when the absolute minimum of the function $t \mapsto a+b p(t)$ has the value 0 , in which case this density has the value $1 / 2$. Our second result concerns the transition of the density from 0 to 1 through $1 / 2$, i.e. when passing through the critical line. A third result concerns square Hill's equation with $p(t)=\operatorname{sign}(\cos (2 \pi t))$, an example where the function $p$ is discontinuous; this example exhibits dramatic differences with the Morse case.

We briefly outline this paper. First, in section 1.1 we introduce the asymptotic radial stability density as a function of the direction and present the main result; for the proofs see 


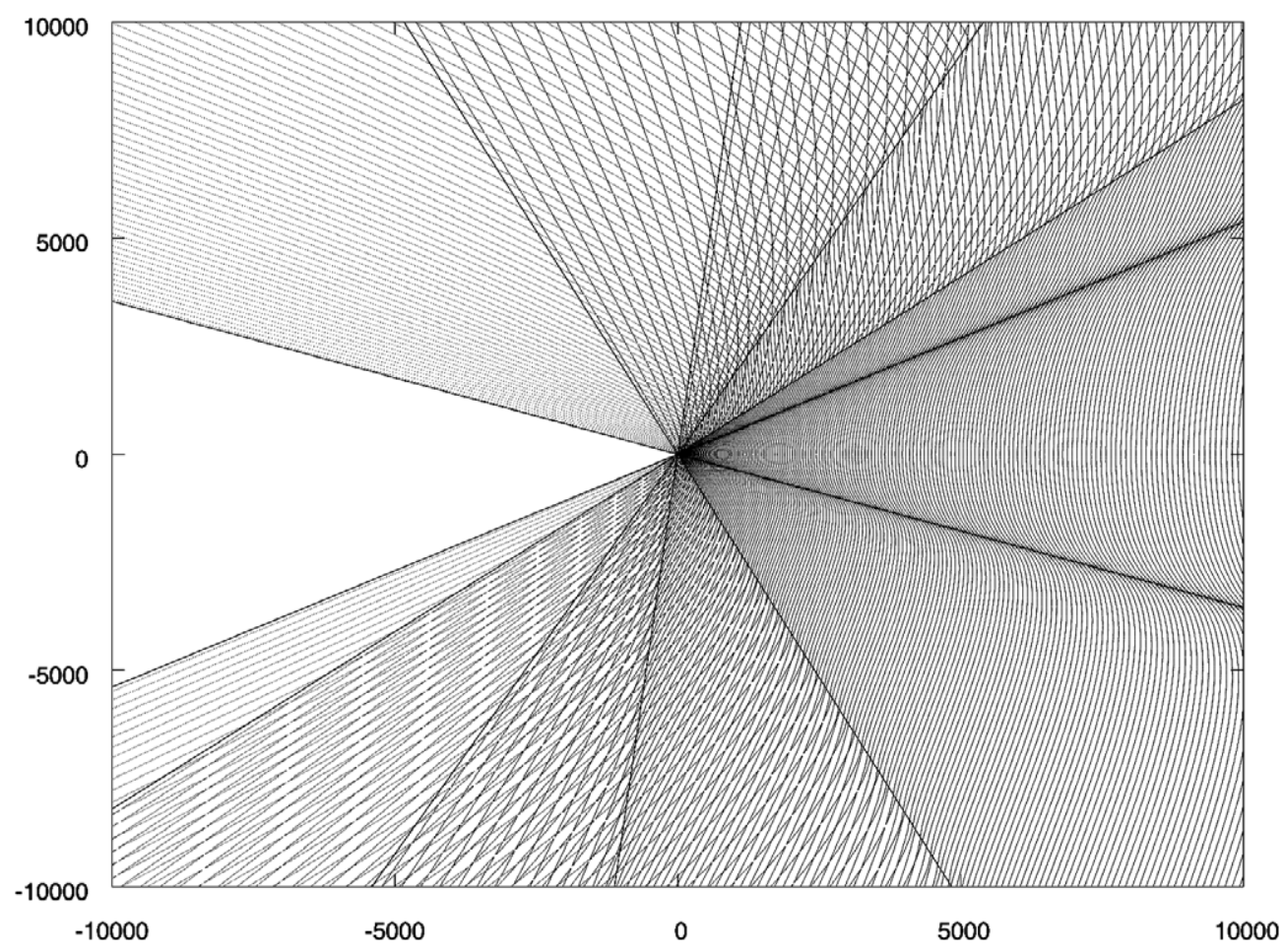

Figure 3. Bifurcation diagram for a non-reversible Hill equation for the choice $p(t)=\cos (t)+$ $\cos (2 t)+\cos (3 t-1)$. One can distinguish 12 different regions. The lines marking the transition from one region to another correspond to values of $b / a$ such that $a+b p(t)$ has an extremum whose value equals zero. The two half lines bounding a sector which contains the positive $a$-axis are the critical lines in that example. In the opposite sector there is instability for all $(a, b)$. The three lines with smaller positive slope correspond to minima. The other ones to maxima.

sections 2 and 3. Then, in section 1.2 we formulate our results regarding the transition of the density when moving through the critical line; for the proof see section 4 . In section 1.3 we state the result for the square Hill equation and give a proof in section 5.

\subsection{Asymptotic radial density of the stability domain}

Consider a measurable set $S \subset \mathbb{R}^{2}$ and a ray through the origin in $\mathbb{R}^{2}$ defined by a point $(a, b) \neq(0,0)$. We introduce 'polar coordinates' $\omega$ and $\chi$ through

$$
a=\omega^{2} \cos (\chi), \quad b=\omega^{2} \sin (\chi) .
$$

Let $L(\chi ; k)$ be the line segment of length $k$ lying on the ray, with one end at the origin.

Definition 1 (Asymptotic radial density). The asymptotic radial density of $S$ in the direction $\chi$ is the limit, if it exists,

$$
\rho_{S}(\chi)=\lim _{k \rightarrow \infty} \frac{|S \cap L(\chi ; k)|}{k}
$$

where $|\cdot|$ denotes the Lebesgue measure on the line.

We shall apply definition 1 to the stability domain

$$
S_{p}=\left\{(a, b) \in \mathbb{R}^{2} \mid \text { Hill's equation (2) is stable }\right\},
$$

abbreviating $\rho(\chi)=\rho_{S_{p}}(\chi)$. The main result of this paper then reads as follows. 
Theorem 1 (Asymptotic radial stability density in dependence of the direction). In Hill's equation (2) let $p$ be a $C^{2}$ periodic Morse function with average $\int_{0}^{1} p(t) \mathrm{d} t=0$ and let $p_{m}=\min p(t)$ and $p_{M}=\max p(t)$. Then

$$
\rho(\chi)= \begin{cases}1 & \text { if } \tan (\chi) \text { is inside the interval } I:=\left(-p_{M}^{-1},-p_{m}^{-1}\right), \\ 1 / 2 & \text { if } \tan (\chi) \text { is one of the endpoints of } I, \\ 0 & \text { if } \tan (\chi) \text { is outside } I .\end{cases}
$$

In the following we shall call a direction $\chi$ stable when $\rho(\chi)=1$ and unstable when $\rho(\chi)=0$ and similarly for sectors. We first reformulate the setting of theorem 1 by introducing

$$
q_{\chi}(t)=\cos (\chi)+p(t) \sin (\chi) .
$$

Often we fix the direction $\chi$ and just write $q=q_{\chi}$. We then consider the general Hill equation

$$
\ddot{x}+\omega^{2} q(t) x=0, \quad q(t+1)=q(t)
$$

with one real parameter $\omega$. We now define the stability density $\rho=\rho(q)$ of the set of stable $\omega$ in the same way as above:

$$
\rho(q)=\lim _{\Omega \rightarrow \infty} \frac{1}{\Omega} \mid\{\omega \mid \text { Hill's equation (8) is stable, } 0<\omega<\Omega\} \mid,
$$

where $|\cdot|$ is the one-dimensional Lebesgue measure. With some abuse of notation we used the same letter $\rho$. Note also that in (4) we used the length in the $(a, b)$-plane to define the density while in (9) we use $\omega$. It is an elementary exercise to prove that if one of these densities exists the other also exists and the values coincide. In the following $\rho$ is going to depend on several other parameters as well.

Theorem 2 (Asymptotic radial stability density in a fixed direction). In Hill's equation (8) assume that $q$ is a $C^{2}$ periodic Morse function. Then, for the asymptotic radial stability density $\rho(q)$ we have the following:

$\rho(q)= \begin{cases}1 & \text { if } q(t)>0 \text { for allt } \\ 1 / 2 & \text { if } q(t) \text { is everywhere positive except at one unique zero, } \\ 0 & \text { if } q \text { is negative on some interval. }\end{cases}$

Observe that the unique zero of $q$ necessarily is a non-degenerate minimum, since $q$ is assumed to be Morse. The proof that theorem 1 follows from theorem 2 is left to the reader. In theorem 1 , the density function $\rho(\chi)$ has a discontinuity for $\tan (\chi)$ at the endpoints of $I$. We shall call these directions and the corresponding lines in the $(a, b)$-plane as critical. For details about the fine structure near such critical lines see section 1.2.

\section{Remark 1.}

- One open question is how rapidly the limits in theorems 1 and 2 are approached as $k \rightarrow \infty$, particularly near the critical lines.

- For the application of definition 1 and for some of the results in this paper it is enough that the periodic function $p$ be continuous, although in many places even $L^{1}$ is enough. As an illustration see section 1.3 concerning the case of square Hill's equation.

Our proof of theorem 2 is divided into several parts. In sections 2 and 3 we deal with the first and third cases of (10), subsequently applying adiabatic theory and exponential domination, leaving the second case $\rho=1 / 2$ to section 4 . As additional information, it is an elementary exercise to check that if $q$ is negative everywhere then (8) is unstable. See the beginning of the proof of lemma 2 for details. 
Rewriting a periodic system (2) in the form

$$
\begin{aligned}
& \dot{x}=y, \\
& \dot{y}=-(a+b p(t)) x, \\
& \dot{t}=1
\end{aligned}
$$

allows us to consider planar Poincaré maps over various time intervals. Since the system is linear and Hamiltonian, these maps are both linear and area preserving, compare with [5]. A Poincaré map over the entire period often is called monodromy map. In the case of (8) this map will be denoted by $F_{\omega}$. We recall that stability is equivalent to

$$
\left|\operatorname{Tr} F_{\omega}\right|<2,
$$

in which case the eigenvalues of $F_{\omega}$ are on the complex unit circle without being real, again see [5].

\subsection{Transition of a critical line}

We consider the vicinity of a critical line for the case of (2) where $p$ is a $C^{2}$ periodic Morse function, investigating how the asymptotic radial density of stability varies when passing from a stable sector to an unstable one. As said before a Morse function is understood as a smooth real-valued function with has no degenerate critical points.

Theorem 3 (Width of transition domain). In Hill's equation (2) assume that the $C^{2}$ periodic Morse function $p$ of zero average has a unique absolute minimum at $t=0$ of the local form

$$
p(t)=p_{0}+p_{2} t^{2}+o\left(t^{2}\right),
$$

as $t \rightarrow 0$, with $p_{0}<0$ and $p_{2}>0$. Then, for any given value of the parameter $a>0$, for $b_{\text {crit }}=-a / p_{0}$, the function $t \mapsto a+b p(t)$ has a non-degenerate minimum at $t=0$. Then the transition from the unstable sector to the stable sector takes place in a range

$$
\left|b-b_{\text {crit }}\right|=\mathcal{O}\left(a^{1 / 2}\right)
$$

as $a \rightarrow \infty$.

A similar result holds when $p$ has a unique absolute maximum at $t=0$ and $b<0$.

The next theorem makes this more precise by considering equation (8) where $q$ is a Morse function with a unique absolute minimum at $t=0$ having a weak dependence on $\omega$ : $q(t)=c t^{2}-d / \omega+o\left(t^{2}\right)$ near $t=0$.

Theorem 4 (Transition near a critical line). Consider Hill's equation (8) with a Morse function q possessing a unique local minimum at $t=0$ as described above:

$$
\ddot{x}+\omega^{2}\left(c t^{2}-\frac{d}{\omega}+o\left(t^{2}\right)\right) x=0 .
$$

Then the density of stability as a function of the parameters $(c, d)$ tends to

$$
\rho(c, d)=\frac{1}{\pi} \arccos \left(\tanh \left(\frac{\pi d}{2 \sqrt{c}}\right)\right),
$$

as $\omega \rightarrow \infty$.

These results rest on properties of fundamental solutions of the Airy and Weber (or parabolic cylinder) equations [1]. For proofs see section 4. 


\subsection{The case of square Hill's equation}

We now consider a case where the function $p$ in (2) is discontinuous, namely where

$$
p(t)=\left\{\begin{aligned}
1 & \text { for } 0 \leqslant t \leqslant 1 \\
-1 & \text { for } 1<t \leqslant 2,
\end{aligned}\right.
$$

where we note that $p$ has period 2. The corresponding equation (2) is known as square Hill's equation. The asymptotic radial density of stability $\rho(\chi)=0$ for $\chi>1 / 4 \pi$, but in contrast with the smooth case the transition to the situation where $\rho(\chi)=1$ does not take place in the immediate vicinity of the line $b=a$. As we shall see now the entire sector $1 / 4 \pi>\chi>0$ is needed for the transition to the stable case $\rho=1$.

To be more precise, given that

$$
\frac{b}{a}=\tan \chi
$$

we put $1-\delta=\tan \chi$ and let $\delta$ run over the interval $[0,1]$, thereby considering $\rho=\rho(\delta)$. Let $\omega_{1}$ and $\omega_{2}$ be defined by $\omega_{1}^{2}=a(2-\delta), \omega_{2}^{2}=a \delta$ or, equivalently, $\omega_{1}=\sqrt{a-b}, \omega_{2}=\sqrt{a+b}$. We consider the case $\omega_{1} / \omega_{2}=\sqrt{\tan (\chi+\pi / 4)} \notin \mathbb{Q}$, a condition satisfied in a set of $\chi$-values of full measure.

Theorem 5 (Asymptotic radial density of stability in square Hill's case). In the above circumstance the asymptotic radial density of stability $\rho=\rho(\delta)$ is monotonically increasing in $\delta$, with

$$
\lim _{\delta \downarrow 0} \rho(\delta)=0 \quad \text { and } \quad \lim _{\delta \uparrow 1} \rho(\delta)=1 .
$$

Furthermore, the following asymptotic expansions hold true:

$$
\rho(\delta)=\left\{\begin{array}{l}
\left.\frac{2}{\pi^{2}} \sqrt{2 \delta}\left(\log \left(\frac{1}{\delta}\right)+\mathcal{O}(1)\right) \quad \text { as } \delta \downarrow 0 \text { (i.e. } b \uparrow a\right), \\
1-\frac{4}{\pi^{2}}(1-\delta)+\mathcal{O}\left((1-\delta)^{3} \quad \text { as } \delta \uparrow 1 \text { (i.e. } b \downarrow 0\right) .
\end{array}\right.
$$

Remark 2. We consider the original parameter plane $\mathbb{R}^{2}=\{a, b\}$. From theorem 5 it follows that at the critical line $b=a$, where $\delta=0$, we have $\rho(\delta)=0$. At the line $b=0$, corresponding to $\delta=1$, we have $\rho(\delta)=1$. In between, i.e. for $0<b<a$, always $\rho(\delta)<1$. This is in sharp contrast with the situation described in the regular case of theorem 2.

For a more detailed description, proofs and comparison with the case $\omega_{1} / \omega_{2} \in \mathbb{Q}$ see section 5.

\subsection{Conclusions}

As the warning example of square Hill's equation of section 1.3 already indicates, the regularity of the function $p$ has a strong influence on the transition properties of the asymptotic radial stability density $\rho=\rho(\chi)$.

This study has been complemented by many numerical simulations which show that below to the critical line but close to it, the size of the instability intervals along a line of fixed slope tends to zero as a power of $\varepsilon$ which depends on the regularity of the function $p$. For the analytical case the evidence is that the convergence to zero is $\mathcal{O}(\exp (-c \omega))$ for some $c>0$.

Another amazing fact, which the reader can guess from the bottom part of figure 2 and, especially, in figure 3, is the existence of a 'stability web' in the domain with $\rho=0$. It is 
formed by the domains of stability in the $(a, b)$-plane. Due to the fact that different 'channels of stability' are associated with disjoint sets of the rotation number, the channels are disjoint and they produce a 'zigzagging' structure. The structure of this web is associated with the relative minima/maxima of the functions $q$. The details will be presented in a forthcoming paper.

\subsection{Outline of the proofs}

We present a brief outline of the set-up of our proofs as previously indicated. The proof of theorem 2 is divided into several parts. For the first and third case of (10) see section 2, 'Proof of theorem 2: the elliptic case of positive $q$ ' based on adiabatic theory, and section 3, 'Proof of theorem 2: the dominantly hyperbolic case where $q$ changes sign' based on exponential domination. For the second case see section 4, 'Near tangent zeroes of $q$ ' which uses Airy and Weber functions. The proofs of theorems 3 and 4 can be found in section 4 as well. Finally the set-up and proof of theorem 5 are discussed in section 5, 'Square Hill's equation: a case of discontinuous $q$ '.

\section{Proof of theorem 2: the elliptic case of positive $q$}

We start by proving the first statement of theorem 2 where the function $q$ is positive, using an approach via adiabatic invariance [11]. In general, we can rewrite Hill's equation (8) as a system

$$
\begin{aligned}
& \dot{x}=-\omega y, \\
& \dot{y}=\omega q(t) x .
\end{aligned}
$$

Since our interest is with large $\omega$, we rescale the time via $\omega t=\tau$. Through, the paper we shall use ${ }^{\circ}=\mathrm{d} / \mathrm{d} t$ and $^{\prime}=\mathrm{d} / \mathrm{d} \tau$ and $\varepsilon=1 / \omega$. Derivation with respect to other independent variables will be indicated explicitly. This change recasts (13) into

$$
\begin{aligned}
& x^{\prime}=-y, \\
& y^{\prime}=q(\varepsilon \tau) x .
\end{aligned}
$$

Since $\varepsilon=1 / \omega \ll 1$, this is a slowly varying Hamiltonian system and we make use of adiabatic invariance by introducing the ( $\tau$-dependent) action-angle variables $(I, \theta)$ :

$$
\begin{aligned}
& q^{1 / 4} x=\sqrt{I} \cos \theta \\
& q^{-1 / 4} y=\sqrt{I} \sin \theta
\end{aligned}
$$

the positivity of $q$ is used here. We note that $\mathrm{d} x \wedge \mathrm{d} y=\frac{1}{2} \mathrm{~d} I \wedge \mathrm{d} \theta$, so the Hamiltonian character is preserved. Our system (14) takes the form

$$
\begin{aligned}
I^{\prime} & =\frac{1}{2} \varepsilon \frac{\dot{q}}{q} I \cos 2 \theta, \\
\theta^{\prime} & =\sqrt{q}-\frac{1}{4} \varepsilon \frac{\dot{q}}{q} \sin 2 \theta,
\end{aligned}
$$

where $\dot{q}=\left.(\mathrm{d} / \mathrm{d} t) q(t)\right|_{t=\varepsilon \tau}$. The angle equation is independent of the action variable, as is to be expected from the linearity of the original system. Denoting $\sqrt{q(\varepsilon \tau)}=\Omega(\varepsilon \tau)$ and $\dot{q} / q=\varrho(\varepsilon \tau)$, we rewrite (16) as

$$
\theta^{\prime}=\Omega(\varepsilon \tau)-\varepsilon \varrho(\varepsilon \tau) \sin 2 \theta .
$$



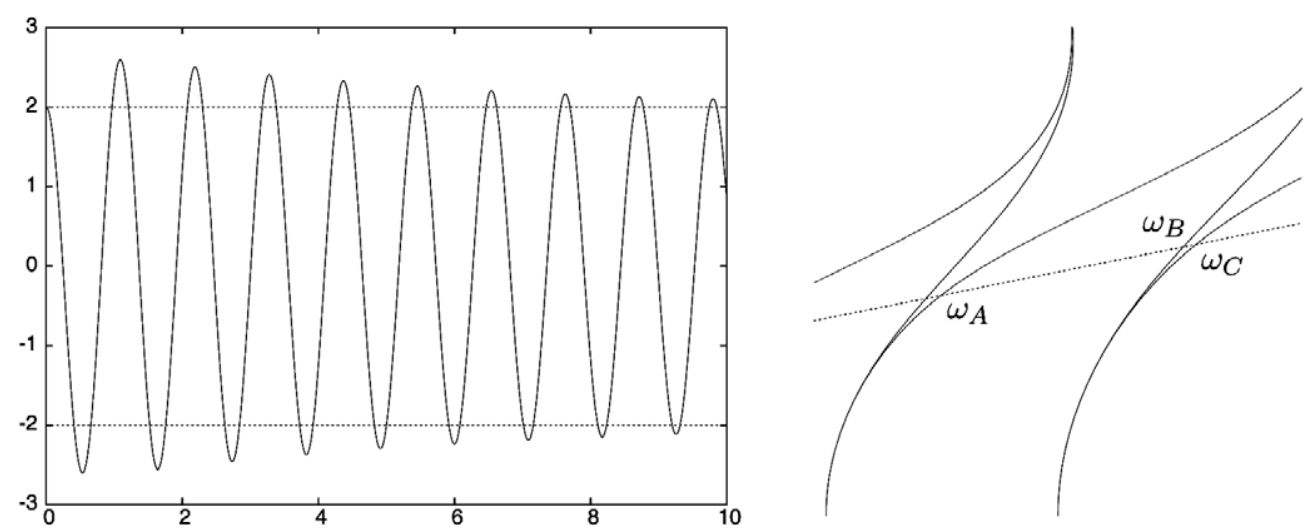

Figure 4. Left: for a line below but close to a critical line the trace $\operatorname{Tr} F_{\omega}$ tends to oscillate periodically in $\omega$, with an amplitude tending to 2. Right: indication of $\omega_{A}$ on the right-hand side of an instability gap and the next gap $\left[\omega_{B}, \omega_{C}\right]$.

It is easy to show (e.g. [5]) that

$$
\theta\left(\frac{1}{\varepsilon}\right)-\theta(0)=\int_{0}^{1 / \varepsilon} \Omega(\varepsilon \tau) \mathrm{d} \tau+\mathcal{O}(\varepsilon)
$$

and, in a similar way, that

$$
I(1 / \varepsilon)-I(0)=\mathcal{O}(\varepsilon) .
$$

From (18) we obtain for the symplectic angle $\psi(t)=\theta(\omega t)$, expressed in terms of the original time and $\omega=1 / \varepsilon$ :

$$
\psi(1)-\psi(0)=\omega \int_{0}^{1} \sqrt{q(t)} \mathrm{d} t+\mathcal{O}\left(\omega^{-1}\right) .
$$

Consider now two values $\omega_{A}<\omega_{B}$ such that the monodromy matrices $F_{\omega}$ with $\omega_{A}<\omega<\omega_{B}$ lie inside a stability domain, with $F_{\omega_{A}}$ and $F_{\omega_{B}}$ on opposite boundaries, see figure 4 . To be specific assume that $\operatorname{Tr} F_{\omega_{A}}=2$ and $\operatorname{Tr} F_{\omega_{B}}=-2$.

Thus

$$
\begin{aligned}
& \psi_{A}(1)-\psi_{A}(0)=2 \pi n, \\
& \psi_{B}(1)-\psi_{B}(0)=(2 n+1) \pi,
\end{aligned}
$$

see, e.g. [13]. From (20) we obtain

$$
\omega_{B}-\omega_{A}=\pi\left(\int_{0}^{1} \sqrt{q} \mathrm{~d} t\right)^{-1}+\mathcal{O}\left(\omega_{A}^{-1}\right) .
$$

Since $\mathcal{O}\left(\omega_{A}^{-1}\right)$ is small and $\int_{0}^{1} \sqrt{q} \mathrm{~d} t=\mathcal{O}\left(\omega^{0}\right)$, it follows that every stability interval is of length $\mathcal{O}\left(\omega^{0}\right)$ for large $\omega$.

To prove that the asymptotic stability density $\rho=1$ it suffices to show that the instability gaps are short for large $\omega$. In the same notation as (21), we have

$$
\psi_{C}(1)-\psi_{C}(0)=(2 n+1) \pi,
$$

compare with (21). 
We now compute the monodromy matrix $F_{\omega}$, taking the initial conditions

$$
\left(\begin{array}{l}
x \\
y
\end{array}\right)=\left(\begin{array}{c}
q_{0}^{-\frac{1}{4}} \\
0
\end{array}\right) \quad \text { and } \quad\left(\begin{array}{c}
0 \\
q_{0}^{-\frac{1}{4}}
\end{array}\right),
$$

where $q_{0}=q(0)$ corresponding to

$$
I=1, \theta=0 \quad \text { and } \quad I=1, \theta=1 / 2 \pi,
$$

respectively. Setting $J=\int_{0}^{1} \sqrt{q(t)} \mathrm{d} t$, we obtain, using (15) and (18), (19):

$$
\begin{aligned}
F_{\omega} & =\left(\begin{array}{cc}
q_{0}^{-1 / 4}(\cos (\omega J)+\mathcal{O}(\varepsilon)) & -q_{0}^{-1 / 4}(\sin (\omega J)+\mathcal{O}(\varepsilon)) \\
q_{0}^{1 / 4}(\sin (\omega J)+\mathcal{O}(\varepsilon)) & q_{0}^{1 / 4}(\cos (\omega J)+\mathcal{O}(\varepsilon))
\end{array}\right)\left(\begin{array}{cc}
q_{0}^{\frac{1}{4}} & 0 \\
0 & q_{0}^{-\frac{1}{4}}
\end{array}\right) \\
& =\left(\begin{array}{cc}
\cos (\omega J) & -q_{0}^{-1 / 2} \sin (\omega J) \\
q_{0}^{1 / 2} \sin (\omega J) & \cos (\omega J)
\end{array}\right)+\mathcal{O}(\varepsilon) .
\end{aligned}
$$

Since $\operatorname{Tr} F_{\omega_{B}}=-2$, it follows that

$$
2 \cos \left(\omega_{B} J\right)+\mathcal{O}(\varepsilon)=-2 .
$$

Inserting $\omega_{B} J=(2 n+1) \pi+\xi$ the latter equation rewrites to

$$
-2\left(1-\frac{\xi^{2}}{2}\right)+\mathcal{O}(\varepsilon)=-2,
$$

which yields that $\xi=\mathcal{O}(\sqrt{\varepsilon})$. Similarly, $\omega_{C}$ is $\mathcal{O}(\sqrt{\varepsilon})$-close to $(2 n+1) \pi$ and therefore

$$
\omega_{C}-\omega_{B}=\mathcal{O}(\sqrt{\varepsilon}) .
$$

This completes the proof of the first part of theorem 2 .

\section{Remark 3.}

- Although instability intervals indeed correspond to unstable $F_{\omega}$ we do not need this in the proof. This instability can be proven from the monotonic dependence of the rotation angle $\psi$ on $\omega$, using the standard Sturm estimate and the positivity of $q$.

- In the setting of theorem 1 , the above proof implies that $\rho=1$ inside the appropriate sector.

- The results of theorem 2 depend on the smoothness of $q$. For example, if $q$ is only $C^{1}$, the $\mathcal{O}(\sqrt{\varepsilon})$-estimate of $(24)$ is a priori only $o(1)$. Some special properties of the differential equations can lead to improved results on averaging.

\section{Proof of theorem 2: the dominantly hyperbolic case where $q$ changes sign}

We now turn to the third part of theorem 2 where $q$ changes sign. Since $q$ is assumed to be a $C^{2}$ periodic Morse function, it changes sign an even number of times, where the graph crosses the zero-level transversally. Without loss of generality we consider the case where $q$ has exactly two such crossings. Our goal is to show that $\rho(q)=0$, which now means that the stability domains are narrow for large $\omega$.

In figure 5 we depict the graph of $q$ under consideration. The monodromy map $F_{\omega}$ is decomposed as

$$
F_{\omega}=C_{u} \circ M_{-} \circ C_{d} \circ M_{+} .
$$

Here $M_{+}, M_{-}$are the strongly elliptic, respectively hyperbolic parts and $C_{u}, C_{d}$ are the signchanges of $q$ in 'upward', respectively 'downward' direction. We recall that these constituting 


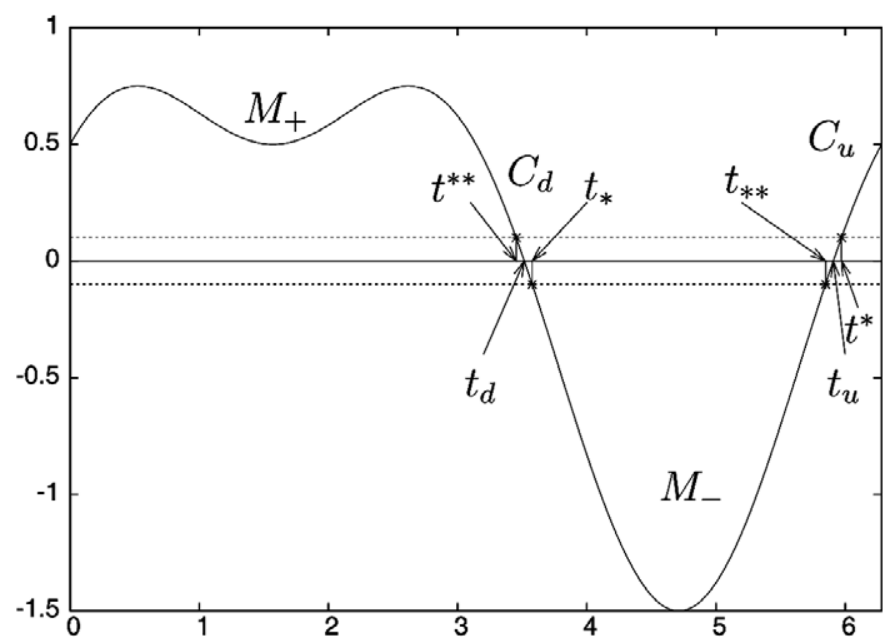

Figure 5. Graph of $q$, in the case of two transversal sign changes. The monodromy map $F_{\omega}$ is decomposed as $F_{\omega}=C_{u} \circ M_{-} \circ C_{d} \circ M_{+}$. Here $M_{+}, M_{-}$are the strongly elliptic, respectively hyperbolic, parts and $C_{u}, C_{d}$ are the transitions through the sign change of $q$ in 'upward', respectively, 'downward' direction.

Poincaré maps are all linear and area preserving. We shall show that the contribution of the strongly hyperbolic part $M_{-}$dominates, which leads to an exponential decay of the stability domains as $\omega \rightarrow \infty$.

For concreteness we assume that $q$ crosses 0 increasing (decreasing) at $t=t_{u}\left(t=t_{d}\right)$. Then values $t^{*}\left(t_{* *}\right)$ and $t_{*}\left(t^{* *}\right)$ are chosen to the right (left) of $t_{u}$ and $t_{d}$, respectively. Hence the matrices $M_{+}, C_{d}, M_{-}, C_{u}$ account, respectively, for the transitions from $t^{*}$ to $t^{* *}$, from $t^{* *}$ to $t_{*}$, from $t_{*}$ to $t_{* *}$ and from $t_{* *}$ to $t^{*}$. The concrete values of $t_{*, * *}$ and $t^{*, * *}$ will be chosen appropriately in section 3.3.

In the following we shall use in many places local approximations of the vectorfield. To take into account the neglected part we rely on the following result, which is an immediate consequence of Gronwall's lemma.

Lemma 1. Consider the differential equation $\dot{w}=f(t, w)+\mu g(t, w)$ on a compact $K \subset$ $\mathbb{R} \times \mathbb{R}^{n}$ with $|\mu| \leqslant \mu_{0}$. Let $\varphi_{\mu}\left(t, t_{0}, w_{0}\right)$ be the flow, denoting as $\varphi_{0}$ the flow if $\mu=0$. Let $L_{f}$ and $L_{g}$ be Lipschitz constants of $f$ and $g$ with respect to $w$, and $\|g\| \leqslant N$. Then $\left\|\varphi_{\mu}\left(t, t_{0}, w_{0}\right)-\varphi_{0}\left(t, t_{0}, w_{0}\right)\right\| \leqslant|\mu| \exp \left(M\left|t-t_{0}\right|\right)$, where $M=\max \left\{L_{f}+\mu L_{g}, N\right\}$.

\section{Remark 4.}

- In anticipation we note that $t_{*, * *}$ and $t^{*, * *}$ are going to be chosen such that the distances to the nearby point $t_{u}, t_{d}$ will be of the form $K_{j} \varepsilon^{2 / 3}$ for suitable large values of $K_{j}, j=u, d$. It will prove convenient that the values of $|q|$ at these four points are all equal and this value is of the form $\hat{q}=\hat{K} \varepsilon^{2 / 3}$ for a very large constant $\hat{K}$.

- The latter can be achieved by choosing $K_{j}$ such that this choice compensates the different slopes of $q$ at $t_{u}, t_{d}$. Note that from lemma 1 it follows that the quadratic and higher order parts of $q$ are irrelevant for small $\varepsilon$.

We shall compute all constituting matrices in the $(x, y)$ coordinates, using $\tau$ as independent variable, compare with (14). Further adaptations of the time variable will be applied in due course and whenever needed. 


\subsection{The strongly elliptic part $M_{+}$}

For the strongly elliptic part $M_{+}$we proceed as in the elliptic case of section 2 where now $M_{+}$ is the Poincaré map from $t=t^{*}$ to $t=t^{* *}$. As mentioned we assume $q\left(t^{*}\right)=q\left(t^{* *}\right)=: \hat{q}$. In this case we define $J=\int_{t^{*}}^{t^{* *}} \sqrt{q(t)} \mathrm{d} t$, then

$$
M_{+}=\left(\begin{array}{cc}
\cos (\omega J)+\mathcal{O}(\varepsilon) & -\hat{q}^{-1 / 2} \sin (\omega J)+\mathcal{O}(\varepsilon) \\
\hat{q}^{1 / 2} \sin (\omega J)+\mathcal{O}(\varepsilon) & \cos (\omega J)+\mathcal{O}(\varepsilon)
\end{array}\right)
$$

Compare with (23). We observe that $J$ is essentially independent of $\omega$, i.e. up to order $\mathcal{O}\left(\omega^{-1}\right)$ as $\omega \rightarrow \infty$. The only mild dependence appears through the variation of $t^{*}, t^{* *}$ with $\omega$, which is of the same order, because integrals like $\int_{t_{u}}^{t^{*}} \sqrt{q(t)} \mathrm{d} t$ are $\mathcal{O}(\varepsilon)$.

\subsection{The strongly hyperbolic part $M_{-}$}

Here we discuss the strongly hyperbolic part $M_{-}$using rather standard arguments. The starting point is system (14)

$$
\begin{aligned}
& x^{\prime}=-y, \\
& y^{\prime}=q(\varepsilon \tau) x,
\end{aligned}
$$

which in polar coordinates $x=r \cos \varphi, y=r \sin \varphi$ take the form

$$
\begin{aligned}
\varphi^{\prime} & =\sin ^{2} \varphi+q(\varepsilon \tau) \cos ^{2} \varphi, \\
r^{\prime} & =r(q(\varepsilon \tau)-1) \sin \varphi \cos \varphi .
\end{aligned}
$$

Lemma 2. Let $M_{-}$be the Poincaré map from $t=t_{*}$ to $t=t_{* *}$ and assume $q\left(t_{*}\right)=q\left(t_{* *}\right)=$ : $-\hat{q}$. Then the following holds true.

- The unstable (respectively stable) eigenvectors of $M_{-}$have polar angles close to $-\sqrt{\hat{q}}$ (respectively $\sqrt{\hat{q}})$, with a relative error $\mathcal{O}\left(\hat{K}^{-1}\right)$ as $\omega \rightarrow \infty$.

- The dominant eigenvalue of $M_{-}$is of the form $\exp \left(c_{*} \omega\right)$ for some $c_{*}>0$, essentially independent of $\omega$, i.e. up to order $\mathcal{O}\left(\omega^{-1}\right)$ as $\omega \rightarrow \infty$.

Proof. We consider the $\varphi$-dynamics between $t_{*}$ and $t_{* *}$, noting that this is independent of $r$. So we can define a one-dimensional Poincaré map $\Phi$ over this interval. Considering the direction of $\varphi$ at the boundaries $\varphi=-\pi / 2,0$, and recalling that $q<0$ in the interval $(-\pi / 4,0)$, it follows by Poincaré-Bendixson like arguments that $\Phi$ has at least one fixed point in $(-\pi / 2,0)$. Along this orbit the derivative $r^{\prime}$ is strictly positive. This gives us an expanding eigenvector. In a similar way we find a contracting eigenvector for initial $\varphi$ in $(0, \pi / 2)$. This also proves the unicity of the eigenvectors.

Along the orbit starting at the fixed point of $\Phi$ in $(-\pi / 2,0)$ the variation of $r$ is given by

$$
r_{\text {final }}=\exp \left(1 / 2 \omega \int_{t_{*}}^{t_{* *}}(q(t)-1) \sin 2 \varphi(t) \mathrm{d} t\right):=\exp \left(c_{*} \omega\right) .
$$

The mild dependence of $c_{*}$ on $\omega$ is due to the small variation of $t_{*}, t_{* *}$ with $\omega$.

On the other hand, the equation for $\varphi$ in (27) forms a slow-fast system, the slow variable being $t$ and the fast one $\varphi$. In the fourth quadrant $\varphi(\tau)$ will closely follow the fixed point of the 'frozen' system, $\varphi(\tau)=-\arctan (-\sqrt{q(\varepsilon \tau)})$. To prove which is the behaviour of the difference between the frozen value and the correct value we analyse its evolution near a point like $t_{u}$. It is not restrictive to assume $t_{u}=0$ and that near $t=0$, the function $q(t)$ is $t$. Slopes 
different from 1 introduce simply a constant factor, and the nonlinear terms shall be considered later.

We introduce a new independent variable $z$ defined by $t=\varepsilon^{2 / 3} z$. Hence

$$
\mathrm{d} \varphi / \mathrm{d} z=\varepsilon^{-1 / 3} \sin ^{2}(\varphi)+\varepsilon^{1 / 3} z \cos ^{2}(\varphi)
$$

giving a frozen value $\varphi^{*}(z)=-\arctan \left(\varepsilon^{1 / 3}(-z)^{1 / 2}\right) \approx-\varepsilon^{1 / 3}(-z)^{1 / 2}$. We define $\chi=$ $\varphi-\varphi^{*}(z)$. The linearization around $\varphi^{*}$ gives

$$
\frac{\mathrm{d} \chi}{\mathrm{d} z}=-2(-z)^{1 / 2} \chi-\frac{\varepsilon^{1 / 3}}{2(-z)^{1 / 2}}
$$

We must study the value of $\chi$ starting at a value $z=-M$ when we reach a value $z=-K$ with $K$ very large and $M \gg K$. Note that the effect of the nonlinear terms in $q(t)$ and the ones missing in (29) can be bounded by changing the factors 2 and $1 / 2$ in (29) by nearby values.

The solution of the linear equation (29) at $z=-K$ is given by

$$
\chi(-K)=C-\varepsilon^{1 / 3} \int_{-M}^{-K} \frac{d z}{2(-z)^{1 / 2}} \exp \left(\frac{4}{3}\left((-K)^{3 / 2}-(-z)^{3 / 2}\right)\right)
$$

where $C=\chi(-M) \exp \left(\frac{4}{3}\left((-K)^{3 / 2}-(-M)^{3 / 2}\right)\right)$, which can be neglected for arbitrary values of $\chi(-M)$. The integral can be computed by successive integration by parts to obtain an expansion in powers of $K^{-1}$ (some of the exponents are half integers) and whose first term is $1 /(4 K)$. This proves the claim about the eigenvectors.

We conclude that vectors of the forms $(1,-\sqrt{\hat{q}})^{t}$ and $(1, \sqrt{\hat{q}})^{t}$ under $M_{-}$are mapped to the vectors $\mathrm{e}^{c_{*} \omega}(1,-\sqrt{\hat{q}})^{t}$ and $\mathrm{e}^{-c_{*} \omega}(1, \sqrt{\hat{q}})^{t}$, respectively. This gives for $M_{-}$the expression

$$
M_{-}=\left(\begin{array}{cc}
\cosh \left(c_{*} \omega\right) & -\hat{q}^{-1 / 2} \sinh \left(c_{*} \omega\right) \\
-\hat{q}^{1 / 2} \sinh \left(c_{*} \omega\right) & \cosh \left(c_{*} \omega\right)
\end{array}\right)
$$

up to a relative error $\mathcal{O}\left(\hat{K}^{-1}\right)$. Note that the format of (30) is quite similar to (26).

\subsection{Near transversal sign change of $q$}

Next we turn to the Poincaré maps $C_{u}$ and $C_{d}$ corresponding to the transversal sign changes of $q$, see figure 5. We first consider the case of $C_{u}$, which we shall relate to an Airy equation of the form

$$
\frac{\mathrm{d}^{2} w}{\mathrm{~d} z^{2}}+z w=0
$$

compare with [1, section 10.4], where we changed the sign of the independent variable $z$.

Without loss of generality and for simplicity we assume that $t_{u}=0$ and let $c=\dot{q}(0)>0$. We now introduce $t_{*}=\delta$, where $\delta$ will be determined appropriately. We start by discussing orders of magnitude, considering the angular equation

$$
\theta^{\prime}=q^{1 / 2}-\frac{\varepsilon}{4} \frac{\dot{q}}{q} \sin 2 \theta
$$

of (16). In view of the adiabatic theory, see section 2 , we need that $q^{1 / 2} \gg \varepsilon \dot{q} / q$, which implies that $t^{1 / 2} \gg \varepsilon t^{-1}$, meaning that we have to choose $\delta \gg \varepsilon^{2 / 3}$. Therefore we now specify $\delta=K \varepsilon^{2 / 3}$, with $K \gg 1$ fixed. Note that $q\left(t_{*}\right)=c K \varepsilon^{2 / 3}\left(1+\mathcal{O}\left(\varepsilon^{2 / 3}\right)\right)$ which can be set equal to $\hat{K} \varepsilon^{2 / 3}$ with $\hat{K}$ large and fixed, by a suitable choice of $K$. Let $\Pi_{\delta}$ be the Poincaré map from $\tau=0$ to $\tau=\omega \delta$. 
Lemma 3. Assume that $q$ is of class $C^{2}$. For $\delta=K \varepsilon^{2 / 3}$, where $\varepsilon \ll 1$ (but with fixed $\left.K \gg 1\right)$, one has

$$
\Pi_{\delta}=\left(\begin{array}{cc}
f\left(c^{1 / 3} K\right) & -(\varepsilon c)^{-1 / 3} g\left(c^{1 / 3} K\right) \\
-(\varepsilon c)^{1 / 3} \frac{d f}{d z}\left(c^{1 / 3} K\right) & \frac{d g}{d z}\left(c^{1 / 3} K\right)
\end{array}\right)+\mathcal{O}\left(\varepsilon^{2 / 3}\right)
$$

Here $f(z), g(z)$ are the solutions of (31) with the conditions $f(0)=1, \frac{d f}{d z}(0)=0, g(0)=$ $0, \frac{d g}{d z}(0)=1$. They can be related to the standard Airy functions $\mathrm{Ai}(-z), \mathrm{Bi}(-z)$ in the oscillatory domain, see [1]. Note that the (real) argument $z=c^{1 / 3} K$ of $f, g$ (or of $\mathrm{Ai}, \mathrm{Bi}$ ) is not at all small, so that we could use asymptotic information for large values if we were interested in more concrete estimates. At this moment this will not be necessary for our purposes.

Remark 5. If we write $q(t)=c t+\mathcal{O}\left(t^{2}\right)$, the effect of the $\mathcal{O}\left(t^{2}\right)$-terms is included in the estimate $\mathcal{O}\left(\varepsilon^{2 / 3}\right)$ of (32). This follows from lemma 1.

Proof. We must scale the case of $x^{\prime \prime}+c \varepsilon \tau x=0$ to the Airy equation (31). To this end we scale the time as $z=(c \varepsilon)^{1 / 3} \tau$. Then we recall that the final value of $\tau$ in the considered interval is given by $\omega \delta=K \varepsilon^{-1 / 3}$. From this it follows a final value of $c^{1 / 3} K$ for $z$.

The factors $(\varepsilon c)^{ \pm 1 / 3}$ in (32) are due to the change of independent variable from $z$ to $\tau$, and the signs from the definition of $y$ in (14).

Once more note that the terms $(\varepsilon c)^{ \pm 1 / 3}$ can be replaced by $\hat{q}^{ \pm 1 / 2}$ at the price of introducing $\left(c^{1 / 3} K\right)^{1 / 2}$ multiplying $g$ and its inverse multiplying $\mathrm{d} f / \mathrm{d} z$. That is, introducing these factors the format of (32) becomes

$$
\Pi_{\delta}=\left(\begin{array}{cc}
a_{1,1} & \hat{q}^{-1 / 2} a_{1,2} \\
\hat{q}^{1 / 2} a_{1,2} & a_{2,2}
\end{array}\right),
$$

where the functions $a_{i, j}$ only depend only on $c^{1 / 3} K$.

In a completely similar way one can study the Poincaré map $\Pi_{-\delta}$ from $\tau=0$ to $\tau=-\omega \delta$. $\Pi_{-\delta}$ will depend on the exponential part $\mathrm{Ai}(z), \mathrm{Bi}(z)$. In Bi $(z)$ a large factor $\exp \left(\frac{2}{3} z^{3 / 2}\right)$ shows up, however, we recall that $c^{1 / 3} K$ must be taken large but bounded.

The Poincaré map $C_{u}$ is related to $\Pi_{\delta}$ and $\Pi_{-\delta}$ as follows:

$$
C_{u}=\Pi_{\delta} \circ\left(\Pi_{-\delta}\right)^{-1} .
$$

A similar result holds for the Poincaré map $C_{d}$. If the slopes at $t_{u}$ and $t_{d}$ were opposite, $c_{d}=\dot{q}\left(t_{d}\right)=-\dot{q}\left(t_{u}\right)=-c_{u}$ then there would be a simple relation between $C_{u}$ and $C_{d}$. Unfortunately, we cannot assume this in a general, non-reversible, case. Keeping the same value of $|q|$ at all the end points of the intervals requires $c_{u} K_{u}=c_{d} K_{d}=\hat{K}$ which implies that $c_{u}^{1 / 3} K_{u} \neq c_{d}^{1 / 3} K_{d}$ in general. For further use we here present the form of $C_{u}$ and $C_{d}$

$$
C_{u}=\left(\begin{array}{cc}
x_{1} & \hat{q}^{-1 / 2} x_{2} \\
\hat{q}^{1 / 2} y_{1} & y_{2}
\end{array}\right), \quad C_{d}=\left(\begin{array}{cc}
u_{1} & \hat{q}^{-1 / 2} u_{2} \\
\hat{q}^{1 / 2} v_{1} & v_{2}
\end{array}\right),
$$

where the values of $x_{i}, y_{i}, i=1,2$ depend only on $c_{u}^{1 / 3} K_{u}$ and the values of $u_{i}, v_{i}, i=1,2$ depend only on $c_{d}^{1 / 3} K_{d}$. 


\subsection{Narrow stability domains}

We now complete the proof of theorem 2 in the case where the Morse function $q$ changes sign. Harvesting from (26), (30) and (35) we obtain the Poincaré map $F_{\omega} \in S L(2, \mathbb{R})$

$$
F_{\omega}=C_{u} \circ M_{-} \circ C_{d} \circ M_{+} .
$$

as given in (25). First we observe that the product of matrices with the format of (33) always keeps this format. This implies that the factors $\hat{q}^{ \pm 1 / 2}$ do not show up in the trace $\operatorname{Tr} F_{\omega}$. The latter can also be seen by introducing a new time $\sigma:=\hat{q}^{1 / 2} \tau$, which cancels all the occurrences of $\hat{q}^{ \pm 1 / 2}$ in these matrices.

The product of left three matrices $C_{u} \circ M_{-} \circ C_{d}$ in (25) is given by

$$
\left(\begin{array}{ll}
C\left(x_{1} u_{1}+x_{2} v_{1}\right)+S\left(-x_{2} u_{1}-x_{1} v_{1}\right) & C\left(x_{1} u_{2}+x_{2} v_{2}\right)+S\left(-x_{2} u_{2}-x_{1} v_{2}\right) \\
C\left(y_{1} u_{1}+y_{2} v_{1}\right)+S\left(-y_{2} u_{1}-y_{1} v_{1}\right) & C\left(y_{1} u_{2}+y_{2} v_{2}\right)+S\left(-y_{2} u_{2}-y_{1} v_{2}\right)
\end{array}\right) .
$$

A direct computation, keeping only the dominant part $\exp \left(c_{*} \omega\right)$ in $C=\cosh \left(c_{*} \omega\right)$ and $S=\sinh \left(c_{*} \omega\right)$ and also neglecting the relative error terms which are at most $\mathcal{O}\left(\varepsilon^{1 / 3}, \hat{K}^{-1}\right)$, gives

$$
\operatorname{Tr} F_{\omega}=\exp \left(c_{*} \omega\right)(A \cos (\omega J)+B \sin (\omega J)),
$$

where $A$ and $B$ are independent of $\varepsilon$. Furthermore

$$
A^{2}+B^{2}=\left(\left(x_{1}-x_{2}\right)^{2}+\left(y_{1}-y_{2}\right)^{2}\right) \times\left(\left(u_{1}-v_{1}\right)^{2}+\left(u_{2}-v_{2}\right)^{2}\right) .
$$

The expression in (37) cannot be zero because of the symplectic character of $C_{u}$ and $C_{d}$. Note that the effect of the relative errors can be included as tiny changes in the value of $c_{*}$. Alternatively (36) can be written as

$$
\operatorname{Tr} F_{\omega}=\sqrt{A^{2}+B^{2}} \exp \left(c_{*} \omega\right) \cos \left(\omega J+\psi_{0}\right) .
$$

At the stability boundaries we have

$$
\operatorname{Tr} F_{\omega}= \pm 2, \text { or } \cos \left(\omega J+\psi_{0}\right) \simeq \exp \left(-c_{*} \omega\right),
$$

leading to the estimate

$$
\omega J+\psi_{0}= \pm \frac{\pi}{2}+\hat{M} \mathrm{e}^{-c_{*} \omega}
$$

for some $\hat{M}$ independent of $\omega$. This shows that the width of the stability domains decreases exponentially as $\omega \rightarrow \infty$, thus proving that $\rho(q)=0$.

\section{Near tangent zeroes of $q$}

In this section, we aim to prove the remaining part of theorem 2 and theorems 3 and 4. We consider the $C^{2}$ periodic Morse function $q$ and the corresponding Hill equation (8)

$$
\ddot{x}=-\omega^{2} q(t) x
$$

near zeroes of $q$ where now, locally, we have $q(t)=d+c t^{2}+o\left(|t|^{2}\right)$ for $c>$ 0 . This means that $t=0$ is a non-degenerate minimum of $q$. Presently we restrict to the case where $q$ is even, which makes (8) reversible. As usual, we invoke lemma 1 to bound the effect of the higher order terms, which can make the equation non-reversible. As in section 3.3 we will have to consider Airy type of differential equations [1]. 


\section{Remark 6.}

- In a similar way we could study cases with

$$
q(t)=d+\left\{\begin{aligned}
-c|t|^{\gamma} & \text { as } t<0, \\
c|t|^{\gamma} & \text { as } t \geqslant 0 .
\end{aligned}\right.
$$

Furthermore, the coefficient $c$ might be different on both sides of $t=0$, both in the case of a minimum and in the crossing case. We shall refrain from considering these extensions. In a few remarks we shall return to this setting.

- Even in less regular cases we do not want to have too wild a behaviour of $\dot{q} / q$. Hence, we shall assume that $|\dot{q}(t)|=\gamma c|t|^{\gamma-1}(1+o(1))$.

Again consider the current version of the monodromy map $F_{\omega}=P_{\delta} \circ M_{+}$, compare with (25), where our interest is to arrive at the present analogue $P_{\delta}$ of the Poincaré maps $C_{u}$ and $C_{d}$, compare with section 3.3. Here we start with the case $t>0$, aiming at a suitable Poincaré map $\Pi_{\delta}$ and where, including the case where $t<0$, we arrive at

$$
P_{\delta}=\Pi_{\delta} \circ\left(\Pi_{-\delta}\right)^{-1},
$$

compare with (34). The evenness over $q$, i.e. that $q(-t) \equiv q(t)$ for (14)

$$
\begin{aligned}
& x^{\prime}=-y, \\
& y^{\prime}=q(t) x
\end{aligned}
$$

implies invariance with respect to the two symmetries

$$
(x, y, t) \mapsto(x,-y,-t) \quad \text { and } \quad(x, y, t) \mapsto(-x, y,-t) .
$$

From this we obtain the following:

Lemma 4 (Reversible Poincaré map near tangency). If

$$
\Pi_{\delta}=\left(\begin{array}{ll}
a_{11} & a_{12} \\
a_{21} & a_{22}
\end{array}\right) \text {, then } \Pi_{-\delta}=\left(\begin{array}{rr}
a_{11} & -a_{12} \\
-a_{21} & a_{22}
\end{array}\right)
$$

and

$$
\begin{aligned}
P_{\delta} & =\left(\begin{array}{ll}
a_{11} & a_{12} \\
a_{21} & a_{22}
\end{array}\right)\left(\begin{array}{ll}
a_{22} & a_{12} \\
a_{21} & a_{11}
\end{array}\right) \\
& =\left(\begin{array}{ll}
a_{11} a_{22}+a_{12} a_{21} & 2 a_{11} a_{12} \\
2 a_{21} a_{22} & a_{11} a_{22}+a_{12} a_{21}
\end{array}\right)
\end{aligned}
$$

Remark 7. In the case where $q$ changes sign we have to proceed as in the previous subsection, namely saying that $\Pi_{\delta}=\Pi_{\delta}(K)$, then $\Pi_{-\delta}=\Pi_{\delta}(-K)$, etc.

Arguing as at the beginning of section 3.3, again consider the angular equation

$$
\theta^{\prime}=q^{1 / 2}-\frac{\varepsilon}{4} \frac{\dot{q}}{q} \sin 2 \theta
$$

of (16). The fact that we want $q^{1 / 2} \gg \varepsilon \dot{q} / q$ implies that $t \gg \varepsilon t^{-1}$, meaning that we have to choose $\delta \gg \varepsilon^{1 / 2}$. Note that in that case for $t=\delta$ we have

$$
\omega q^{1 / 2}=\varepsilon^{-1 / 2} \text {. }
$$

Therefore we now specify $\delta=K \varepsilon^{1 / 2}$, again with $K \gg 1$ fixed. In the $\tau$ variable the interval will be of length $K \varepsilon^{-1 / 2}$. 
We now have to scale the case of $\ddot{x}=-\omega^{2} q(t) x$ with $q(t)=d+c t^{2}$ to a generalized (and shifted) Airy equation

$$
\frac{\mathrm{d}^{2} w}{\mathrm{~d} z^{2}}=-\left(D+z^{2}\right) w
$$

with the fundamental solutions

$$
\begin{aligned}
& f_{D}(z) \text { with initial conditions } w(0)=0, \frac{\mathrm{d} w}{\mathrm{~d} z}(0)=1, \\
& g_{D}(z) \text { with initial conditions } w(0)=1, \frac{\mathrm{d} w}{\mathrm{~d} z}(0)=0 .
\end{aligned}
$$

Note that $f_{1,0}(z)=f(z)$ and $g_{1,0}(z)=g(z)$. This approach leads to the following generalization of lemma 3 .

Lemma 5. Assume that $q$ is a $C^{2}$ periodic Morse function which near an absolute minimum located at $t=0$ behaves as $q(t)=d+c t^{2}(1+o(1))$. Then for a fixed constant $K \gg 1$ and $\delta=K \varepsilon^{1 / 3}$ the following holds true:

$$
\Pi_{\delta}=\left(\begin{array}{cc}
f_{D}(z) & -\left(\varepsilon^{2} c\right)^{-1 / 4} g_{D}(z) \\
-\left(\varepsilon^{2} c\right)^{1 / 4} \frac{\mathrm{d} f_{D}}{\mathrm{~d} z}(z) & \frac{\mathrm{d} g_{D}}{\mathrm{~d} z}(z)
\end{array}\right)(1+o(1)),
$$

as $\varepsilon \downarrow 0$, where all functions in the matrix are evaluated at $z=c^{1 / 4} K$ and where $d$ and $D$ are related by $d=D\left(\varepsilon^{2} c\right)^{1 / 2}$.

Proof. We want to reduce (14) with $q(\varepsilon \tau)=d+c|\varepsilon \tau|^{2}$ to (39), skipping the higher order terms whose effect is taken into account later using lemma 1. Writing $z=\alpha \tau$ it follows that one has to take

$$
\alpha=\left(\varepsilon^{2} c\right)^{1 / 4}
$$

and the value of $D$ as stated in the lemma.

The final value of $z$ will be $\alpha K \varepsilon^{-1 / 2}=c^{1 / 4} K$.

Notice that the format of the matrix is like in (33) with the entries $a_{i, j}$ depending only on $c^{1 / 4} K$.

As a consequence of lemmas 4 and 5 the following dominant part is found

$$
\operatorname{Tr} P_{\delta}=2\left(f_{D} \frac{\mathrm{d} g_{D}}{\mathrm{~d} z}+g_{D} \frac{\mathrm{d} f_{D}}{\mathrm{~d} z}\right)\left(c^{1 / 4} K\right)
$$

so here we do need asymptotic information on the behaviour of Airy functions (and their generalizations) near $\infty$.

At this point we note that equation (39) for large positive $D$ it is mainly elliptic and for large negative $D$ mainly hyperbolic. The same is true for the monodromy matrix $F_{\omega}=P_{\delta} \circ M_{+}$. Hence, when $D$ makes the transition from positive large to negative large, the density $\rho$ will pass from close to 1 to close to 0 . According to lemma 5 this happens for variations of $d$ which are of the order of $\varepsilon$ as $\varepsilon \downarrow 0$. For equation (2) this can be expressed in terms parameters $(a, b)$ in the form of theorem 3 .

\section{Remark 8.}

- Given $D$ the value of $\operatorname{Tr} P_{\delta}$, according to information the equation (39), tends to oscillate in a sinusoidal way for increasing $\delta$. Let $A(D)$ be the limit amplitude. Then $\operatorname{Tr} F_{\omega}$ behaves like $A(D) \sin (\omega J+\psi)$ for some phase $\psi$. It follows that the value of $\rho$ along the line associated with $D$, close to a critical line tends to

$$
\rho(D)=\frac{2}{\pi} \arcsin \left(\frac{2}{A}\right) \text {. }
$$


- A slight modification of the above leads to a generalization of theorem 3 into the setting of remark 6 that runs as follows: in Hill's equation (2) assume $p$ has an unique absolute minimum at $t=0$ and that $p(t)=m+n|t|^{\gamma}$ locally, with $m>0, n>0, \gamma>0$. Then for any fixed value of the parameter $a$, for $b_{\text {crit }}=-a / m$, the function $t \mapsto a+b p(t)$ has a minimum at $t=0$. Moreover, then the transition from the unstable sector to the stable sector takes place in a range $\left|b-b_{\text {crit }}\right|=\mathcal{O}\left(a^{2 /(2+\gamma)}\right)$.

- The case of $\gamma \neq 2$ concerns more degenerate minima of the function $q$. It is of interest to see what is the effect of degeneracies and possible unfoldings. In particular, we can consider local models like $q(t)=t^{4}+\alpha_{2} t^{2}+\alpha_{1} t+\alpha_{0}$.

\subsection{Exactly at a critical line}

We want to be more precise about the behaviour at a critical line, when the $C^{2}$ Morse function $q \geqslant 0$ has a unique minimum with value 0 attained, say, at $t=0$. The aim of this section is to prove the remaining part of theorem 2, which asserts that the asymptotic radial stability density equals $1 / 2$.

Proof of theorem 2, critical case. To compute the monodromy map $F_{\omega}$ for the critical case we split the full domain in a neighbourhood of the minimum and the remainder domain. In the latter case we essentially are dealing with a rotation over an angle going to $\infty$ with $\omega$, compare with section 2. Again compare with the discussion at the beginning of section 3.3 and consider the angular equation of (16), where we aim to ensure that the first term $\sqrt{q}$ is dominant. Therefore we consider an interval around $t=0$ of size $K \varepsilon^{1 / 2}$ (or $K \varepsilon^{-1 / 2}$ in the $\tau$ variable, with fixed large $K$, as discussed before.

On the other hand, there exist $c_{1}, c_{2}$ as close to $c$ as needed, such that $c_{1}|t|^{\gamma} \leqslant q(t) \leqslant c_{2}|t|^{\gamma}$ in that interval, provided that $\omega$ is sufficiently large. The variations in $q$ play a minor role in the amplitude and phase of the local solutions. Hence we may well consider that in the interval around $t=0$ the equation is exactly $\ddot{x}+\omega^{2} c t^{2} x=0$. Higher order effects, including a possible non-reversibility, are included in the previous bounds. Scaling $t$ we can assume that we are dealing with the following variation:

$$
\frac{\mathrm{d}^{2} x}{\mathrm{~d} s^{2}}+s^{2} x=0
$$

of the Airy equation, which is reversible, again compare with [1]. Later we shall return to the suitable scalings. First consider $s \geqslant 0$. Let $x_{1}(s), x_{2}(s)$ be the fundamental solutions with

$$
x_{1}(0)=1, \quad \frac{\mathrm{d} x_{1}}{\mathrm{~d} s}(0)=0 \quad \text { and } \quad x_{2}(0)=0, \quad \frac{\mathrm{d} x_{2}}{\mathrm{~d} s}(0)=1 .
$$

A direct computation gives

$$
x_{1}(s)=\sum_{k \geqslant 0}\left[-\frac{1}{4}\left(\frac{s^{2}}{2}\right)^{2}\right]^{k} \frac{\Gamma\left(\frac{3}{4}\right)}{k ! \Gamma\left(k+\frac{3}{4}\right)}=\Gamma\left(\frac{3}{4}\right)\left(\frac{z}{2}\right)^{-v_{1}} J_{\nu_{1}}(z)
$$

and

$$
x_{2}(s)=t \sum_{k \geqslant 0}\left[-\frac{1}{4}\left(\frac{s^{2}}{2}\right)^{2}\right]^{k} \frac{\Gamma\left(\frac{5}{4}\right)}{k ! \Gamma\left(k+\frac{5}{4}\right)}=\Gamma\left(\frac{5}{4}\right)\left(\frac{z}{2}\right)^{-\nu_{2}} J_{\nu_{2}}(z),
$$

where $J_{v}$ denote the Bessel function of the first kind, with $v_{1}=-\frac{1}{4}, v_{2}=\frac{1}{4}$ and where $z=s^{2} / 2$. 
Using Hankel's asymptotic expansions for $J_{v}(z)$ for large $z$ (see, e.g. [1, section 9.2]: $J_{v}(z) \approx \sqrt{(2 / \pi z)} \cos (z-(\pi / 4)-(\nu \pi / 2))+\mathcal{O}\left(z^{-3 / 2}\right)$, and taking (14) into account, we obtain

$$
\begin{aligned}
x_{1}(s) & \approx \Gamma\left(1-\frac{1}{4}\right) \kappa \frac{1}{\sqrt{\pi}} s^{-1 / 2} \cos (z-\delta), \\
y_{1}(s)=-\frac{\mathrm{d} x_{1}}{\mathrm{~d} s}(s) & \approx \Gamma\left(1-\frac{1}{4}\right) \kappa \frac{1}{\sqrt{\pi}} s^{1 / 2} \sin (z-\delta), \\
x_{2}(s) & \approx-\Gamma\left(\frac{1}{4}\right) \kappa^{-1} \frac{1}{\sqrt{\pi}} s^{-1 / 2} \sin (z+\delta), \\
y_{2}(s)=-\frac{\mathrm{d} x_{2}}{\mathrm{~d} s}(s) & \approx \Gamma\left(\frac{1}{4}\right) \kappa^{-1} \frac{1}{\sqrt{\pi}} s^{1 / 2} \cos (z+\delta),
\end{aligned}
$$

where $z$ is as before, $\kappa=\sqrt{2}$ and $\delta=\frac{\pi}{8}$.

As in the proof of lemma 5 we scale from $s$ to $\tau$ as $s=\alpha \tau, \alpha=\left(\varepsilon^{2} c\right)^{1 / 4}$. Let

$$
M(s)=\left(\begin{array}{cc}
x_{1}(s) & \alpha^{-1} x_{2}(s) \\
\alpha y_{1}(s) & y_{2}(s)
\end{array}\right)
$$

be the fundamental matrix containing the elements from (42), where the terms depending on $\alpha$ express that we are working in the $(x, y, \tau)$ coordinates. Then the matrix $N=M(s) M(-s)^{-1}$ related to the passage from $-s$ to $s$ is given by

$$
N=\left(\begin{array}{cc}
x_{1} y_{2}+x_{2} y_{1} & 2 \alpha^{-1} x_{1} x_{2} \\
2 \alpha y_{1} y_{2} & x_{1} y_{2}+x_{2} y_{1}
\end{array}\right),
$$

compare with lemma 4. Using (42) and recalling the well-known formula

$$
\Gamma(1-\zeta) \Gamma(\zeta)=\frac{\pi}{\sin (\pi \zeta)}
$$

we obtain the following expressions for the dominant part of the elements of $N$, using that $\sin \frac{\pi}{4}=1 / 2 \sqrt{2}$ :

$$
\begin{array}{ll}
n_{1,1}=n_{2,2} & =-\sqrt{2} \cos (2 z), \\
n_{2,1} & =\alpha \sqrt{2} s(\sin (2 z)-\sin (2 \delta)), \\
n_{1,2} & =-\alpha^{-1} \sqrt{2} s^{-1}(\sin (2 z)+\sin (2 \delta)) .
\end{array}
$$

In this way we know the contribution to the monodromy from some $-s$ to $s$. We recall that the final value of $s$ is $c^{1 / 4} K$. Note that if we stop computations here, that is, we do not take into account the effect of the elliptic part, the maximal trace of $N$ when we allow $z$ to change is

$$
\frac{2}{\sin \left(\frac{\pi}{4}\right)}=2 \sqrt{2} \text {. }
$$

We shall see that the role of this elliptic part is not so relevant. The effect of the complementary interval was given by matrix $M_{+}$in (26). We recall that the value of $\hat{q}^{1 / 2}$ now is $c^{1 / 2} K \varepsilon^{1 / 2}$.

From the product $F_{\omega}=M_{+} N$ we obtain

$$
\begin{aligned}
\sin \left(\frac{\pi}{4}\right) \operatorname{Tr} F_{\omega}= & 2 \cos (\omega J) \cos (2 z) \\
& -\sin (\omega J)\left[P(\sin (2 z)-\sin (2 \delta))+P^{-1}(\sin (2 z)+\sin (2 \delta))\right],
\end{aligned}
$$

when only considering dominant terms, where $P=\alpha s \hat{q}^{-1 / 2}$.

From the definitions of $\alpha, s$ and $\hat{q}$ it turns out that $P=1$. Hence we obtain as the final expression for the trace

$$
\operatorname{Tr} F_{\omega}=2 \sqrt{2} \cos (\omega J+2 z)
$$


From (45) it follows that the fraction of values of $\omega$ for which $\left|\operatorname{Tr} F_{\omega}\right|<2$ is given by

$$
\frac{\theta}{\pi / 2} \text { where } \sin (\theta)=1 / 2 \sqrt{2}, \text { hence } \theta=\frac{\pi}{4} \text {. }
$$

This yields the desired value $\rho=1 / 2$ at a critical line.

This concludes the proof of theorem 2 and hence also of theorem 1 .

\section{Remark 9.}

- In the setting of remark 6 the above proof can be modified to obtain the critical density $\rho=2 /(\gamma+2)$.

\subsection{The transition across a critical line}

We now prove theorem 4 regarding the transition across a critical line. Instead of (39) we here use as model the Weber equation

$$
\frac{\mathrm{d}^{2} w}{\mathrm{~d} z^{2}}+\left(\frac{1}{4} z^{2}-a\right) w=0
$$

which leads to parabolic cylinder functions. As usual the local effect of higher order terms $o\left(z^{2}\right)$ in (46) is a negligible correction according to lemma 1 . Let $w_{1}(z), w_{2}(z)$ be fundamental solutions with initial conditions

$$
w_{1}(0)=1, \quad \frac{\mathrm{d} w_{1}}{\mathrm{~d} z}(0)=0, \quad \text { and } \quad w_{2}(0)=0, \quad \frac{\mathrm{d} w_{2}}{\mathrm{~d} z}(0)=1 .
$$

The 'standard' solutions (see [1, sections 19.16, 19.17, 19.21]) are given by

$$
W(a, \pm z)=2^{-3 / 4}\left(\sqrt{\frac{G_{1}}{G_{3}}} w_{1}(z) \mp \sqrt{\frac{2 G_{3}}{G_{1}}} w_{2}(z)\right),
$$

where $G_{1}=|\Gamma(1 / 4+\mathrm{i} a / 2)|, G_{2}=|\Gamma(3 / 4+\mathrm{i} a / 2)|$. The dominant terms of the asymptotic expansions for fixed $a$ and large $z$ then read

where

$$
W(a, z)=\sqrt{\frac{2 \kappa}{z}} \cos (\Phi), \quad W(a,-z)=\sqrt{\frac{2}{\kappa z}} \sin (\Phi),
$$

$$
\Phi=\frac{1}{4} z^{2}-a \log (z)+\frac{\pi}{4}+\frac{1}{2} \phi_{2}
$$

and $\kappa, \phi_{2}$ are functions of $a$ defined by

$$
\kappa=\sqrt{1+\exp (2 \pi a)}-\exp (\pi a), \quad \phi_{2}=\arg \Gamma(1 / 2+\mathrm{i} a),
$$

taking the continuous determination of the argument which gives $\phi_{2}=0$ for $a=0$.

Again using lemma 4 and recalling that in the matrices we are using $-\mathrm{d} w / \mathrm{d} z$ instead $\mathrm{d} w / \mathrm{d} z$, the Poincaré map for the passage from $-z$ to $z$ for large $z$ is given by $N=$ $\left(\begin{array}{ll}n_{1,1} & n_{1,2} \\ n_{2,1} & n_{2,2}\end{array}\right)$, where

$$
\begin{aligned}
& n_{1,1}=n_{2,2}=\frac{1}{2}\left(\kappa+\frac{1}{\kappa}\right) \sin (2 \Phi) \\
& n_{2,1}=\frac{z}{4}\left[\kappa-\frac{1}{\kappa}-\left(\kappa+\frac{1}{\kappa}\right) \cos (2 \Phi)\right] \\
& n_{1,2}=\frac{1}{z}\left[\kappa-\frac{1}{\kappa}+\left(\kappa+\frac{1}{\kappa}\right) \cos (2 \Phi)\right]
\end{aligned}
$$



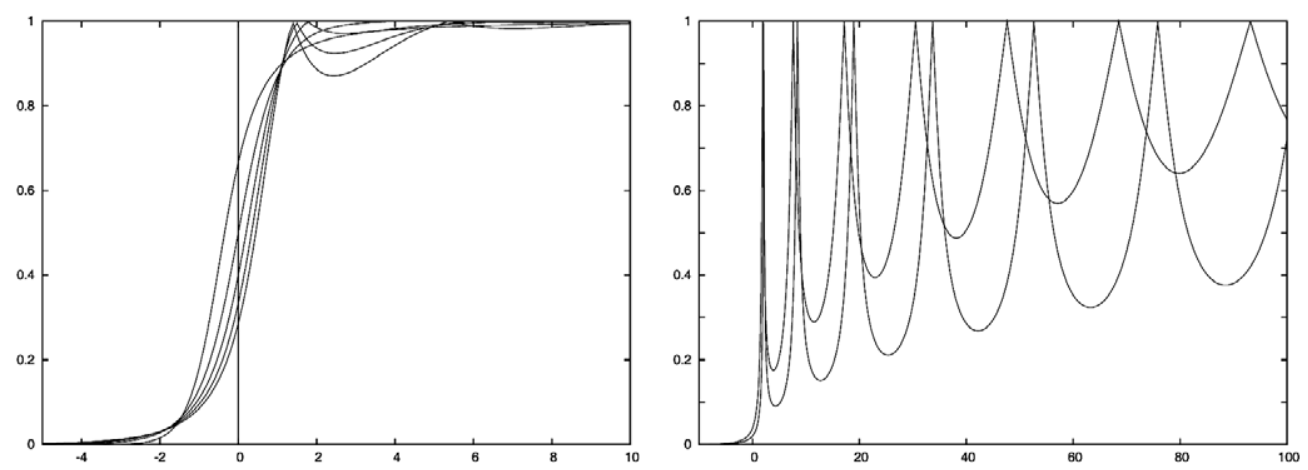

Figure 6. Left: the transition functions, showing the variation of $\rho$ corresponding to different $\gamma$ for (39) as a function of $D$. The values of $\gamma$ when crossing $D=0$ are $1,2,3,4,5$, from top to bottom. The value 2 corresponds to theorem 4. Right: similar plots for $\gamma=50,100$.

Using the same kind of reasoning as in the proof in the previous subsection we also have:

- For the matrix $N$ in (47) the maximal trace when $z$, and therefore $\Phi$, is allowed to vary, is $\kappa+1 / \kappa=2 \sqrt{1+\exp (2 \pi a)}$.

- The effect of the change of independent time from $z$ to $\tau$, affecting $n_{1,2}, n_{2,1}$ is irrelevant for the trace.

- After the scalings the final value $z^{*}$ of $z$ is of the form $(4 c)^{1 / 4} K$. Hence $\Phi=\Phi^{*} \simeq$ $\frac{1}{2} c^{1 / 2} K^{2}$.

- Considering $F_{\omega}=M_{+} N$ the format of the trace $\operatorname{Tr} F_{\omega}$ in that case is similar to (45)

$$
\operatorname{Tr} F_{\omega}=2 \sqrt{1+\exp (2 \pi a)} \sin \left(\omega J+2 \Phi^{*}\right) .
$$

Again the stability density associated with the parameter $a$ in (46) is given by

$$
\rho(a)=\frac{2}{\pi} \arcsin \left(\frac{1}{\sqrt{1+\exp (2 \pi a)}}\right)=\frac{1}{\pi} \arccos (\tanh (\pi a)) .
$$

For any fixed value of $a$ the transition size in $b$ from $\rho$ close to 1 to $\rho$ close to 0 , is $\mathcal{O}\left(a^{1 / 2}\right)=\mathcal{O}(\omega)$. Hence we can write out present Hill's equation as

$$
\ddot{x}+\omega^{2}\left(c t^{2}-\frac{d}{\omega}\right) x=0 .
$$

We have to relate it to the parameter $a$ in (46). A simple scaling gives $a=d c^{-1 / 2} / 2$.

This finishes our proof of theorem 4.

Remark 10. It follows from (48) that $\rho$ is a monotone increasing function of $-a$. One may ask if the same holds for other values of $\gamma$. Unfortunately a closed formula like (48) is not available. It would require explicit solutions for $q$ as mentioned in Remark 6, a kind of fully generalized and shifted Airy equation or some equivalent Weber-like equation with $|z|^{\gamma}$ replacing $z^{2}$ in (46), which would extend the results in [1]. Numerical evidence, obtained independently with these Weber-like equations and by direct estimate of the density for large $\omega$, shows that monotonicity holds only for $\gamma \leqslant 2$. For $\gamma>2$ the density $\rho$ reaches the value $\rho=1$ for a set of positive values of $-a$, see figure 6 . This set seems to be countable. Associated with these values of $-a$ instability pockets $[8,9]$ are detected for reversible systems. 


\section{Square Hill's equation: a case of discontinuous $q$}

The discontinuous case is dramatically different from the smooth cases discussed before. We again refer to Keller et al [12,21,22], Arnold [3, 4, 5] and to [2, 8, 9], restricting to the case of square Hill's equation. So we turn to the discussion of section 1.3 which describes the transition behaviour of the asympotic radial density in the case of square Hill's equation (2) with

$$
p(t)=\left\{\begin{aligned}
1 & \text { for } 0 \leqslant t \leqslant 1, \\
-1 & \text { for } 1<t \leqslant 2,
\end{aligned}\right.
$$

as already defined in (11). In this section, we shall prove theorem 5 and will give some further information on this interesting case where many things can be computed explicitly.

Given that

$$
\frac{b}{a}=\tan \chi
$$

we recall that $1-\delta=\tan \chi$, where $\delta$ runs over the interval $[0,1]$, thereby considering $\rho=\rho(\delta)$. The Poincaré map is

$$
F_{\delta}=\left(\begin{array}{cc}
\cos \omega_{2} & -\omega_{2}^{-1} \sin \omega_{2} \\
\omega_{2} \sin \omega_{2} & \cos \omega_{2}
\end{array}\right)\left(\begin{array}{cc}
\cos \omega_{1} & -\omega_{1}^{-1} \sin \omega_{1} \\
\omega_{1} \sin \omega_{1} & \cos \omega_{1}
\end{array}\right),
$$

where $\omega_{1}^{2}=a(2-\delta)=a+b$ and $\omega_{2}^{2}=a \delta=a-b$ and therefore

$$
\operatorname{Tr} F_{\delta}=2 \cos \omega_{1} \cos \omega_{2}-\left(\frac{\omega_{1}}{\omega_{2}}+\frac{\omega_{2}}{\omega_{1}}\right) \sin \omega_{1} \sin \omega_{2} .
$$

We are interested in the stability of $F_{\delta}=F_{\delta}(a)$ for fixed $\delta$ and $a \rightarrow \infty$. Then, inserting

$$
B=1 / 2\left(\frac{\omega_{1}}{\omega_{2}}+\frac{\omega_{2}}{\omega_{1}}\right)=\frac{1}{\sqrt{\delta(2-\delta)}},
$$

we note that $2 B(\delta)$ attains a minimum 2 at $\delta=1$ and that $\lim _{\delta \downarrow 0} B(\delta)=\infty$. For a better understanding of the asymptotic radial stability density

$$
\rho(\delta)=\lim _{a \rightarrow \infty} \frac{1}{a}\left|\left\{\alpha \in \mathbb{R}|| \operatorname{Tr} F_{\delta}(\alpha) \mid<2,0<\alpha<a\right\}\right|
$$

we consider the case where the ratio $\omega_{1}: \omega_{2}$ is irrational, invoking Birkhoff's ergodic theorem [6], which yields that

$$
\rho(\delta)=\frac{1}{4 \pi^{2}} \lambda\left(\left\{\left(\theta_{1}, \theta_{2}\right) \in \mathbb{T}^{2}|| \operatorname{Tr} F_{\delta}\left(\theta_{1}, \theta_{2}\right) \mid<2\right\}\right) .
$$

Here $\lambda$ is the Lebesgue measure on $\mathbb{T}^{2}$ and

$$
\operatorname{Tr} F_{\delta}\left(\theta_{1}, \theta_{2}\right)=2\left(\cos \theta_{1} \cos \theta_{2}-B(\delta) \sin \theta_{1} \sin \theta_{2}\right) .
$$

To compute the measure in (49) we consider $\operatorname{Tr} F_{\delta}\left(\theta_{1}, \theta_{2}\right)$ as a function on $\mathbb{T}^{2}$.

First, a brief computation shows that the critical points of $\frac{1}{2} \operatorname{Tr} F_{\delta}\left(\theta_{1}, \theta_{2}\right)$ are arranged as follows on the torus.

\begin{tabular}{r|r|r|r|r|l} 
& 0 & $\frac{\pi}{2}$ & $\pi$ & $\frac{3 \pi}{2}$ & $\theta_{2} \rightarrow$ \\
\hline 0 & 1 & - & -1 & - & \\
\hline$\frac{\pi}{2}$ & - & $-B$ & - & $B$ & \\
\hline$\pi$ & -1 & - & 1 & - & \\
\hline$\frac{3 \pi}{2}$ & - & $B$ & - & $-B$ & \\
\hline$\theta_{1}$ & & & & & \\
$\downarrow$ & & & & &
\end{tabular}



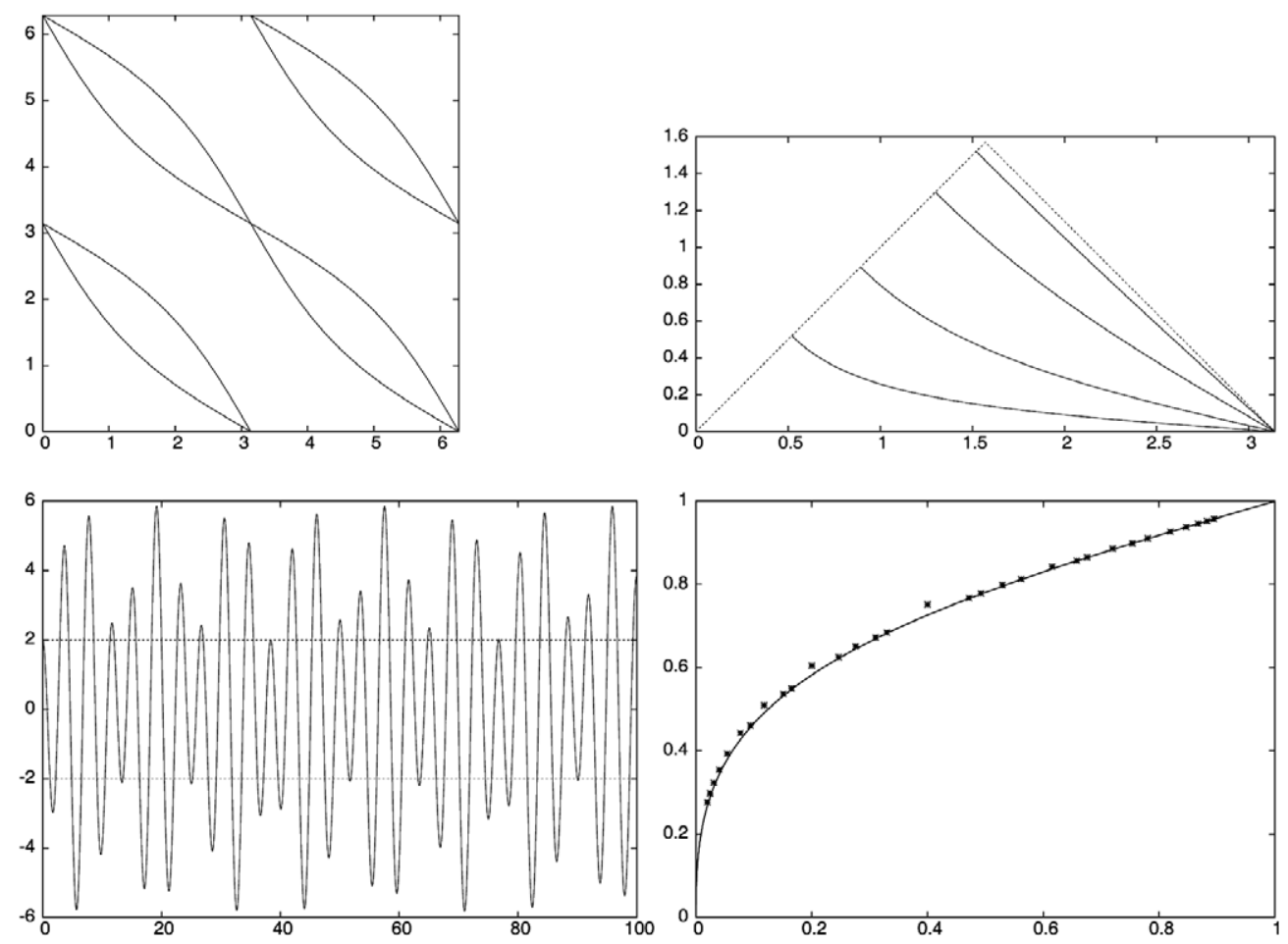

Figure 7. Top left: stability boundaries $\operatorname{Tr} F_{\delta}\left(\theta_{1}, \theta_{2}\right)= \pm 2$. The area inside the 'lenses' corresponds to instability, i.e. where $|\operatorname{Tr}|>2$. Top right: the curves $\mathcal{B}$ displayed in $1 / 16$ of $\mathbb{T}^{2}$ for $\delta=0.9,0.5,0.1,0.01$ from top to bottom. Bottom left: behaviour of the trace $\operatorname{Tr} P$ as a function of $\sqrt{a}$ for $\delta=0.06$. A quasi-periodic pattern is easily seen. Compare with the regularity in figure 4. Bottom right: plot of $\rho(\delta)$ for $\omega_{2} / \omega_{1}$ irrational. For completeness also the values for $\omega_{2} / \omega_{1}=n / m, m \leqslant 10,0<n<m$ are depicted. Then $\delta$ has to be equal to $2 n^{2} /\left(m^{2}+n^{2}\right)$.

Our next concern is with the boundary curves $\operatorname{Tr} F_{\delta}\left(\theta_{1}, \theta_{2}\right)= \pm 2$, see figure 7 . Because of the symmetries it is enough to restrict ourselves to $\frac{1}{16}$ th part of $\mathbb{T}^{2}$, namely to the region bounded by the 'lines'

$$
\ell_{1}=\left\{\theta_{1}=0\right\}, \quad \ell_{2}=\left\{\theta_{2}=\theta_{1}\right\} \quad \text { and } \quad \ell_{3}=\left\{\theta_{1}+\theta_{2}=\pi\right\} .
$$

Inside region (50) the boundary is formed by the curve $\mathcal{B}$, given by $\operatorname{Tr} F_{\delta}\left(\theta_{1}, \theta_{2}\right)=-2$. The value of $\rho$ then is the fraction of area of that region bounded by $\mathcal{B}$ and $\ell_{1}, \ell_{2}$. A summary of the behaviour of $\rho$ is given by theorem 5 in section 1.3 .

Proof of theorem 5. Let $\Delta=\theta_{1}+\theta_{2}, \Sigma=\theta_{1}-\theta_{2}$. Then the condition for $\mathcal{B}$ reads $(1+B) \cos (\Sigma)=-2+(B-1) \cos (\Delta)$. When $\delta \uparrow 1$ and $B \downarrow 1$ we have that $\cos (\Sigma) \downarrow-1$. Hence $\mathcal{B}$ tends to $\ell_{3}$ and therefore $\rho \uparrow 1$. On the other hand, if $\delta \downarrow 0$ and $B \uparrow \infty$, we have $\cos (\Sigma) \rightarrow \cos (\Delta)$. Hence, $\theta_{2} \rightarrow 0$, the boundary $\mathcal{B}$ tends to $\ell_{1}$ and $\rho \downarrow 0$.

To see the monotonicity we consider a line $\Delta=$ ctant. Along it one has $(B+$ $1)^{2}(\mathrm{~d} \cos (\Sigma) / \mathrm{d} B)=2(1+\cos (\Delta))>0$. Therefore $\cos (\Sigma)$ increases with $B$ and then $\Sigma$ decreases. It follows that the curve $\mathcal{B}$ moves monotonically from $\ell_{3}$ to $\ell_{1}$ when $\delta$ decreases.

For the first estimate we compute the area bounded by $\ell_{1}, \ell_{2}$ and $\mathcal{B}$. Now it is better to express $\theta_{2}$ along $\mathcal{B}$ as a function of $\theta_{1}$, starting at the point on $\ell_{2}$, for which $\theta_{1}=\theta_{1}^{*} \simeq \sqrt{2 / C}$. The dominant term is $\theta_{2}=\frac{1}{C} \cot \left(\theta_{1} / 2\right)$. From this the estimate follows. This can be expressed 


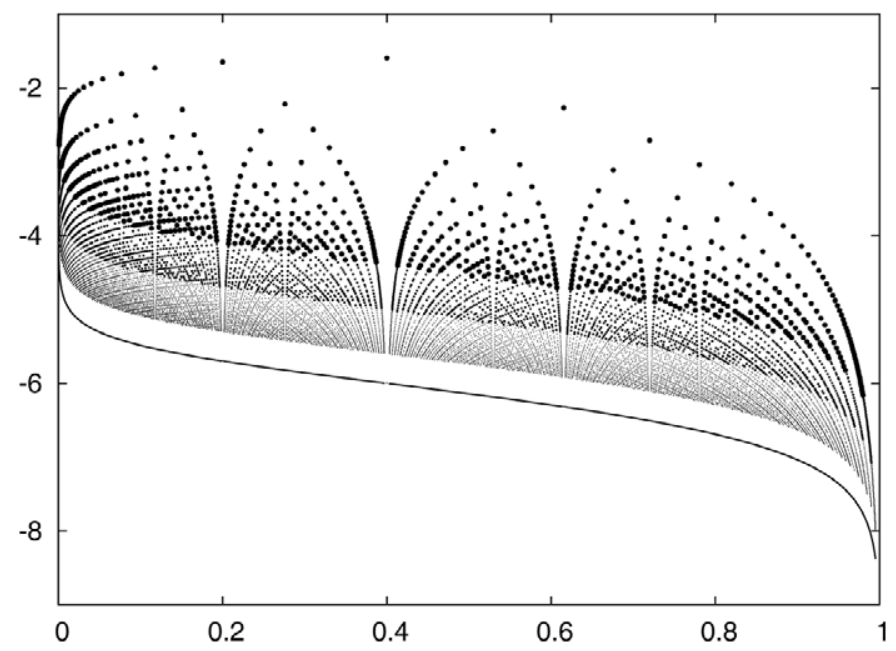

Figure 8. $\log _{10}\left(\rho_{\text {rational }}(\delta)-\rho(\delta)\right)$ as a function of $\delta$ for the values of $\delta$ for which $\omega_{2} / \omega_{1}=$ $n / m \in \mathbb{Q}, 2 \leqslant m \leqslant 200$. The values of $m \leqslant 50$ (respectively $50<m \leqslant 100$; respectively $100<m \leqslant 200$ ) are plotted using large (respectively medium size; respectively small) dots. One can check that, around a give value of $\delta$, the difference is of order $\mathcal{O}\left(\mathrm{m}^{-2}\right)$. This can be checked even better in the lower line which displays $\log _{10}\left(\rho_{\text {rational }}(\delta)-\rho(\delta)\right)+2 \log _{10}(m)-5$.

in terms of $\delta$ for $\delta \rightarrow 0$ as

$$
\rho=\frac{2}{\pi^{2}} \sqrt{2 \delta}\left(\log \left(\frac{1}{\delta}\right)+\mathcal{O}(1)\right) .
$$

For the second asymptotic estimate we compute the area bounded by $\ell_{3}, \ell_{2}$ and $\mathcal{B}$. Using the variables $\Delta$ and $\Sigma$ and $C=B-1$ it is given by

$$
\frac{1}{2} \int_{0}^{\pi} \arccos \left(\frac{2-C \cos (\Delta)}{C+2}\right) d \Delta .
$$

From this the estimate follows easily. As before this can be expressed in terms of $\delta$ as

$$
\rho=1-\frac{4}{\pi^{2}}(1-\delta)+\mathcal{O}\left((1-\delta)^{3}\right),
$$

showing that close to $b=0$ the density $\rho$ is close to linear in $b$.

This finishes our proof of theorem 5 .

The definite integral above has been used to compute data for the plot in figure 7 (bottom right). One can question which is the behaviour for $\omega_{2} / \omega_{1}=n / m \in \mathbb{Q},(n, m)=$ $1,0<n<m$, that is, the density $\rho_{\text {rational }}(\delta)$ for the corresponding values of $\delta$. We write $\theta_{1}=m z, \theta_{2}=n z$. It is enough to check the intervals $z \in[0,2 \pi]$ for which $\left|\operatorname{Tr} F_{\delta}\left(\theta_{1}, \theta_{2}\right)\right|<2$. After some simple manipulation this amounts to compute the zeroes of $(m / n+n / m) T_{m+n}(w)-(m / n+n / m-2) T_{m}(w) T_{n}(w)= \pm 2$, where $T_{k}$ denotes the $k$ th Chebyshev polynomial. Then one can compute $\arccos \left(w_{i}\right)$ for all the zeroes, add the lengths of the intervals for which $|\operatorname{Tr}|<2$ and obtain the desired density along the corresponding line.

It is interesting to note that the values of $\rho_{\text {rational }}(\delta)$ in all cases exceed the ones of $\rho(\delta)$ for the same value of $\delta$. This can be expected because the curves $\mathcal{B}$ shown in figure 7 top right have positive second derivative. Figure 8 displays, in $\log _{10}$ scale, the difference $\rho_{\text {rational }}(\delta)-\rho(\delta)$. 


\section{Acknowledgments}

H W B thanks the Departament de Matemàtica Aplicada i Anàlisi of the Universitat de Barcelona and the Department of Mathematics of Pennsylvania State University for hospitality during various visits. The research of $\mathrm{M} \mathrm{L}$ has been supported in part by the NSF Grant DMS0605878. C S wants to thank the Johann Bernoulli Institute for Mathematics and Computer Science, University of Groningen, for hospitality in different visits. His research has been partially supported by grants MTM2006-05849/Consolider and MTM2010-16425 (Spain) and 2009 SGR-67 (Catalonia).

\section{References}

[1] Abramowitz M and Stegun I A 1965 Handbook of Mathematical Functions (New York: Dover)

[2] Afsharnejad Z 1986 Bifurcation geometry of Mathieu's equation Indian. J. Pure Appl. Math. 17 1284-308

[3] Arnold V I 1983 Remarks on the perturbation theory for problems of Mathieu type Russ. Math. Surv. 38 215-33

[4] Arnold V I 1983 Geometrical Methods in the Theory of Ordinary Differential Equations (Berlin: Springer)

[5] Arnold V I 1978 Mathematical Methods of Classical Mechanics (Graduate Texts in Mathematics vol 60) (New York: Springer)

[6] Arnold V I and Avez A A 1967 Problèmes Ergodiques de la Méchanique Classique (Paris: Gauthier-Villars)

[7] Broer H W 2012 Resonance and fractal geometry Acta Appl. Math. 120 61-86

[8] Broer H W and Levi M 1995 Geometrical aspects of stability theory for Hill's equations Arch. Ration. Mech. Anal. $131225-40$

[9] Broer H W and Simó C 2000 Resonance tongues in Hill's equations: a geometric approach J.Diff. Eqns $166290-327$

[10] Broer H W, Puig J and Simó C 2003 Resonance tongues and instability pockets in the quasi-periodic HillSchrödinger equation Commun. Math. Phys. 241 467-503

[11] Levi M 1981 Adiabatic invariants of the linear Hamiltonian systems with periodic coefficients J.Diff. Eqns 42 47-71

[12] Levy D M and Keller J B 1963 Instability intervals of Hill's equation Commun. Pure Appl. Math. 16 469-79

[13] Magnus W and Winkler S 1979 Hill's Equation (New York: Dover)

[14] Mathieu E 1868 Mémoire sur le mouvement vibratoire dune membrane de forme elliptique J. Math. Pures Appl. 13 137-203

[15] Meixner J and Schäfke F W 1954 Mathieusche Funktionen und Sphäroidfunktionen (Berlin: Springer)

[16] van der Pol B and Strutt M J O 1928 On the stability of the solutions of Mathieu's equation Phil. Mag. 5 18-38 (7 th Ser.)

[17] Pöschel J and Trubowitz E 1986 Inverse Spectral Theory (New York: Academic)

[18] Puig J and Simó C 2006 Analytic families of reducible linear quasi-periodic differential equations Ergodic Theory Dyn. Syst. 26 481-524

[19] Puig J and Simó C 2011 Resonance tongues in the quasi-periodic Hill-Schrödinger equation with three frequencies Reg. Chaotic Dyn. 16 62-79

[20] Stoker J J 1950 Nonlinear Vibrations in Mechanical and Electrical Systems (New York: Interscience)

[21] Weinstein M I and Keller J B 1985 Hill's equation with a large potential SIAM J. Appl. Math. 45 954-8

[22] Weinstein M I and Keller J B 1987 Asymptotic behavior of stability regions for Hill's equation SIAM J. Appl. Math. 47 941-58 